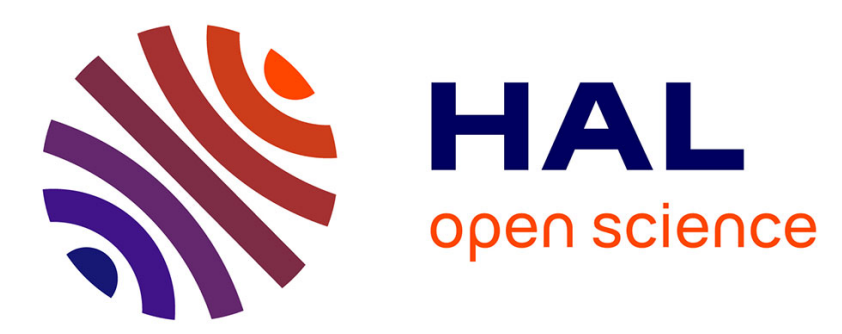

\title{
On the justification of topological derivative for wave-based qualitative imaging of finite-sized defects in bounded media
}

\author{
Marc Bonnet
}

\section{- To cite this version:}

Marc Bonnet. On the justification of topological derivative for wave-based qualitative imaging of finitesized defects in bounded media. Engineering Computations, 2022, 39 (1), pp.313-336. 10.1108/EC08-2021-0471 . hal-03319821

\section{HAL Id: hal-03319821 \\ https://hal.science/hal-03319821}

Submitted on 13 Aug 2021

HAL is a multi-disciplinary open access archive for the deposit and dissemination of scientific research documents, whether they are published or not. The documents may come from teaching and research institutions in France or abroad, or from public or private research centers.
L'archive ouverte pluridisciplinaire HAL, est destinée au dépôt et à la diffusion de documents scientifiques de niveau recherche, publiés ou non, émanant des établissements d'enseignement et de recherche français ou étrangers, des laboratoires publics ou privés. 


\title{
ON THE JUSTIFICATION OF TOPOLOGICAL DERIVATIVE FOR WAVE-BASED QUALITATIVE IMAGING OF FINITE-SIZED DEFECTS IN BOUNDED MEDIA
}

\author{
MARC BONNET
}

\begin{abstract}
.
Purpose This work contributes to the general problem of justifying the validity of the heuristic that underpins medium imaging using topological derivatives (TDs), which involves the sign and the spatial decay away from the true anomaly of the TD functional. We consider here the identification of finite-sized (i.e. not necessarily small) anomalies embedded in bounded media and affecting the leading-order term of the acoustic field equation.
\end{abstract}

Design/methodology/approach TD-based imaging functionals are reformulated for analysis using a suitable factorization of the acoustic fields, which is facilitated by a volume integral formulation. The three kinds of TDs (single-measurement, full-measurement and eigenfunctionbased) studied in this work are given expressions whose structure allows to establish results on their sign and decay properties. The latter are obtained using analytical methods involving classical identities on Bessel functions and Legendre polynomials, as well as asymptotic approximations predicated on spatial scaling assumptions.

Findings The sign component of the TD imaging heuristic is found to be valid for multistatic experiments and if the sought anomaly satisfies a bound (on a certain operator norm) involving its geometry, its contrast and the operating frequency. Moreover, upon processing the excitation and data by applying suitably-defined bounded linear operatirs to them, the magnitude component of the TD imaging heuristic is proved under scaling assumptions where the anomaly is small relative to the probing region, the latter being itself small relative to the propagation domain. We additionally validate both components of the TD imaging heuristic when the probing excitation is taken as an eigenfunction of the source-to-measurement operator, with a focusing effect analogous to that achieved in time-reversal based methods taking place. These findings extend those of earlier studies to the case of finite-sized anomalies embedded in bounded media.

Originality/value The originality of the paper lies in the theoretical justifications of the TDbased imaging heuristic for finite-sized anomalies embedded in bounded media.

Full abstract The concept of topological derivative (TD) is known to provide, through its heuristic interpretation involving its sign and its spatial decay away from the true anomaly, a basis for the qualitative imaging of finite-sized anomalies. The TD imaging heuristic is currently partially backed by conditional mathematical justifications. Continuing earlier efforts towards the justification of TD-based identification, this work investigates the acoustic wave-based imaging of finite-sized (i.e. not necessarily small) medium anomalies embedded in bounded domains and affecting the leading-order term of the acoustic field equation. Both the probing excitation and the measurement are assumed to take place on the domain boundary. We extend to this setting the analysis approach previously used for unbounded media with either refractionindex anomalies and far-field measurements (Bellis et al., Inverse Problems 29:075012, 2013) or mass-density anomalies and meaurements at finite distance (Bonnet, Cakoni, Inverse Problems 35:104007, 2019). Like in the latter work, TD-based imaging functionals are reformulated for analysis using a suitable factorization of the acoustic fields, facilitated by a volume integral formulation. Our results, which echo corresponding results of our earlier investigations, conditionally validate the TD imaging heuristic. Moreover, we show on a geometrically simple configuration that the spatial behavior of the TD associated with standard $L^{2}$ cost functionals is degraded by "echoes" of the true anomaly, an aspect specific to the present bounded-domain framework. This undesirable effect is removed by a combination of (i) post-processing the measurements by application of a suitable integral operator (a treatment introduced by Ammari et al., 2011, for the analysis of TD-based imaging involving true flaws modelled using smallanomaly asymptotics), and (ii) expressing the background field as an incoming single-layer potential defined in the full space (after an idea used in Bonnet, Cakoni, 2019). Finally, we also show that selecting eigenfunctions of the source-to-measurement operator as excitations enhances the spatial decay properties of the TD functionals.

Date: August 13, 2021.

Key words and phrases. Helmholtz equation, topological derivative, imaging. 


\section{INTRODUCTION}

Inverse scattering has undergone intense investigation over the last quarter century, in particular due to the growth and flourishing of qualitative methods which provide robust and computationally effective alternatives to more traditional approaches based on successive linearizations or PDE-constrained optimization, see Kirsch and Grinberg (2008), Cakoni and Colton (2014), Cakoni et al. (2016) for exposition and references. Qualitative anomaly imaging methods typically involve sampling a spatial region of interest with points $\boldsymbol{z}$ at which an imaging function $W$ is evaluated. In particular, (generalized) linear sampling methods and factorization methods work along those lines and are backed by rigorous mathematical justifications.

The concept of topological derivative (TD) provides an alternative basis for qualitative flaw imaging. The TD of an objective functional $\mathcal{J}$ quantifies the leading perturbation to $\mathcal{J}$ caused by a trial anomaly of vanishingly small radius $\varepsilon$ appearing at a given location $\boldsymbol{z}$ in the (defectfree) background propagation medium. On choosing $\mathcal{J}$ as a misfit functional of a form usually employed in PDE-constrained inversion, a sampling apprach may be defined by setting $W(\boldsymbol{z})$ to the value $\mathcal{T}(\boldsymbol{z})$ of the TD of $\mathcal{J}$ at sampling points $\boldsymbol{z}$. Since the sought flaws are deemed to minimize $\mathcal{J}$, an intuitive heuristics for TD-based imaging consists in postulating that the (possibly multiple) flaw is located where $\mathcal{T}(\boldsymbol{z})$ has a negative sign and largest magnitude.

Topological derivatives, initially introduced and formalized as computational aids for topology optimization (Eschenauer et al., 1994; Sokolowski and Zochowski, 1999), have since seen many extensions and applications, see e.g. Novotny et al. (2019a,b,c) and references therein. In particular, it has proved effective for revealing hidden flaws in a variety of inverse scattering situations, see e.g. Bonnet and Guzina (2004), Dominguez et al. (2005), Guzina and Bonnet (2006), Guzina and Chikichev (2007), Ammari et al. (2012), Laurain et al. (2013), Bellis and Bonnet (2013), Le Louër and Rapún (2017), Bonnet (2018), Carpio et al. (2019). In particular, despite the asymptotic nature of the mathematical concept of TD, abundant computational evidence demonstrates its ability to qualitatively identify spatially-extended objects. The underlying objective functional $\mathcal{J}$ often quantifies the misfit between the available data and its model prediction in a least-squares sense, which makes TD-based imaging implementable irrespective of the available data. Moreover, the practical evaluation of $\boldsymbol{z} \mapsto \mathcal{T}(\boldsymbol{z})$ is straightforward and moderately expensive as it can be expressed as a bilinear function of the incident field and an adjoint field associated with $\mathcal{J}$ (see e.g. Céa et al., 2000).

For given objective functional and physical framework, the formulation of $\mathcal{T}(\boldsymbol{z})$ is a clearly defined and mathematically rigorous operation. By contrast, the mathematical justification of the foregoing TD imaging heuristics is still gappy. Theoretical investigations about TD-based imaging began only about a decade ago. Ammari et al. (2012) studied the imaging of a small single scatterer in an acoustic medium, and its stability with respect to medium or measurement noises, with follow-up studies by Ammari et al. (2013) and Wahab (2015) extending this framework to elastodynamics and electromagnetism, respectively. Guzina and Pourahmadian (2015) addressed the high-frequency limiting behavior of a TD imaging functional. The same general problem was addressed for spatially extended anomalies in unbounded acoustic media, considering refraction-index anomalies and far-field measurements (Bellis et al., 2013) and later mass-density anomalies and finitely remote measurements (Bonnet and Cakoni, 2019).

Bellis et al. (2013) and Bonnet and Cakoni (2019) found, in their respective contexts, the magnitude component of the TD imaging heuristic to be valid, and the guaranteed correctness of the sign component subject to an inequality (involving the operating frequency and the obstacle size and contrast) essentially requiring the flaw to be "moderate". Following up on those previous studies, we consider here the TD imaging heuristics for spatially extended anomalies embedded in a bounded acoustic domain where excitations and measurements take place on the domain boundary. Our main aim is to establish conditions under which the usual TD imaging heuristic is valid when the medium being probed is finite. As in our previous studies, TDs are reformulated for analysis using a suitable factorization of the acoustic fields, facilitated by a volume integral formulation. Sign properties are found to conform to corresponding previous results. Our other main contribution consists of an asymptotic study of the decay of $|\mathcal{T}(\boldsymbol{z})|$ 
is studied by applying to the present context the asymptotic approach introduced in Bonnet and Cakoni (2019) where the sampling region is taken large relative to the obstacle diameter but small relative to the domain size. We first analytically show, on a geometrically simple configuration, that the spatial behavior of the TD associated with standard $L^{2}$ cost functionals is degraded by "echoes" of the true anomaly. This observation prompts us to propose a modified TD-based imaging method whereby (i) measurements are post-processed by application of a suitable integral operator (a treatment introduced by Ammari et al., 2012 for the analysis of TD-based imaging of flaws modelled using small-anomaly asymptotics), and (ii) expressing the background field as an incoming single-layer potential defined in the full space (a variation on an idea used in Bonnet and Cakoni, 2019). The decay properties of the resulting modified TDs are found to be similar to those previously established for unbounded media. We additionally show that selecting eigenfunctions of the source-to-measurement operator as excitations enhances the spatial decay properties of both usual and modified versions of the TD functional.

The article is organized as follows. In Section 2, we formulate the scattering problem by means of a volume integral equation employing the acoustic Neumann function of the propagation domain, allowing a symmetric factorization of the source-to-measurement operator. On that basis, explicit expressions for TDs involving symmetric factorizations are derived for the usual leastsquares misfit objective functional with three kinds of data configuration (single-measurement, full-measurement and eigenfunction-based). Then, sign and spatial decay properties of those TDs are established in Section 3. Modified versions of TDs are introduced in Section 4 and shown in Section 5 to have preserved sign properties but improved decay properties relative to their unmodified counterparts of Section 3. Section 6 then offers some general comments and shows how our analysis framework also applies to situations involving refraction-index, anisotropic or inhomogeneous anomalies. Finally, some proofs are collected in Section 7.

\section{Scattering PRoblem And topological DeRivative}

We begin by specifying some notation conventions used throughout this paper. A tensor algebra notation style is used for vectors or tensors in the physical space $\mathbb{R}^{3}$; in particular, expressions such as $\boldsymbol{A} \cdot \boldsymbol{x}$ or $\boldsymbol{B}: \boldsymbol{C}$, symbols '.' and ':' denote single and double inner products, e.g. $(\boldsymbol{A} \cdot \boldsymbol{x})_{i}=\sum_{i} A_{i j} x_{j}$ and $\boldsymbol{B}: \boldsymbol{C}=\sum_{i, j} B_{i j} C_{i j}$, with component indices always referring to an orthonormal frame. The (Euclidean) norm $\sqrt{\boldsymbol{x} \cdot \boldsymbol{x}}$ of a vector $\boldsymbol{x}$ is denoted by $|\boldsymbol{x}|$, whereas $\|\cdot\|$ indicate norms in function spaces or operator norms. Hat symbols over vectors denote collinear unit vectors, e.g. $\hat{\boldsymbol{x}}:=\boldsymbol{x} /|\boldsymbol{x}|$. For some domain or surface $X \subset \mathbb{R}^{3}$, we denote by $f, g \mapsto(f, g)_{X}:=\int_{X} f \bar{g}$ the sesquilinear form associated with the $L^{2}(X)$ scalar product of scalar or tensor complex-valued functions defined in $X$.

2.1. Direct and scattering problem. We consider a homogeneous and isotropic reference (e.g. acoustic) propagation medium occupying a bounded domain $\Omega$ with boundary $\Gamma:=\partial \Omega$. Time-harmonic propagating waves satisfy the Helmholtz equation

$$
-\Delta u-\kappa^{2} u=0 \quad \text { in } \Omega,
$$

where $\kappa=\omega / c$ is the wavenumber expressed in terms of the wave velocity $c$ and operating angular frequency $\omega$, and $u$ is the scalar acoustic pressure. We assume that $\kappa^{2}$ is not an Neumann eigenvalue of the Laplacian in $\Omega$. The medium hosts an unknown anomaly with compact support $B \Subset \Omega$ whose material properties are characterized by a uniform relative scalar contrast $\beta>-1$. Waves propagating in the perturbed medium then satisfy

$$
\begin{gathered}
-\left(\Delta+\kappa^{2}\right) u=0 \quad \text { in } \Omega \backslash \bar{B}, \quad-\left((\beta+1) \Delta+\kappa^{2}\right) u=0 \quad \text { in } B, \\
\left.u\right|_{+}=\left.u\right|_{-},\left.\quad \partial_{n} u\right|_{+}=\left.\beta \partial_{n} u\right|_{-} \quad \text { on } \partial B
\end{gathered}
$$

where $\partial_{n}:=\boldsymbol{n} \cdot \boldsymbol{\nabla}$ is the normal derivative operator and $\left.(\cdot)\right|_{+}$and $\left.(\cdot)\right|_{-}$denote limiting values on $\partial B$ from outside and inside of $B$, respectively (the case of anisotropic anomalies being concisely discussed in Sec. 6.2). To probe the medium, waves are generated in $\Omega$ by applying a Neumann data $g$ on the boundary:

$$
\partial_{n} u=g \quad \text { on } \Gamma \text {. }
$$


For the linear acoustic model, $g$ is proportional to a given normal wall velocity. The background field $u=u[g]$ then solves problem $(2.1,2.3)$, while the perturbed field $u_{B}=u_{B}[g]$ solves problem $(2.2,2.3)$, so that the presence of an anomaly $B$ is revealed by the non-vanishing scattered field $v_{B}[g]:=u_{B}[g]-u[g]$. The identification problem under consideration is to estimate the anomaly location $B$ given the scattered field $v_{\text {obs }}[g]$ measured on $\Gamma$. To facilitate theoretical analysis, we idealize the situation by assuming the data to be noise-free, i.e. $v_{\text {obs }}[g]=v_{B}[g]$. The topological derivative is known to be only mildly sensitive to data noise, as discussed e.g. in Ammari et al. (2012) and, briefly, in Bonnet and Cakoni (2019, Sec. 6.2).

2.2. Objective functional. We formulate the problem of identifying $B$ as the minimization of an objective functional. For a trial anomaly of support $D$, the least-squares functional

$$
\mathcal{J}[g](D):=\frac{1}{2} \int_{\Gamma}\left|v_{D}[g]-v_{\text {obs }}[g]\right|^{2} \mathrm{~d} S,
$$

which in this form corresponds to exploiting a single experiment with applied excitation $g$, is the most common basis for such optimization-based identification.

We also consider experimental situations which can be thought of as the mathematical idealization of a multistatic experiment, where measurements for all possible excitation / response pairs $\left(g, v_{\text {obs }}[g]\right)$ are available. Letting $\left(Y_{m}\right)_{m \in \mathbb{N}}$ denote a Hilbert basis of $L^{2}(\Gamma)$, a suitable objective functional for this case is then

$$
\mathcal{J}_{\Sigma}(D):=\sum_{m \geq 0} \frac{1}{2} \int_{\Gamma}\left|v_{D}\left[Y_{m}\right]-v_{\text {obs }}\left[Y_{m}\right]\right|^{2} \mathrm{~d} S .
$$

For example, $\left(Y_{m}\right)_{m \in \mathbb{N}}$ can be taken as the set of eigenfunctions of the Laplace-Beltrami operator on $\Gamma$ (Cogar, 2020), which are none other than the spherical harmonics if $\Gamma$ is a sphere.

2.3. Asymptotic of the cost functional. The medium is "sampled" by means of trial anomalies of support $B_{\varepsilon}(\boldsymbol{z})=\boldsymbol{z}+\varepsilon \mathcal{B}$ and size $\varepsilon>0$, centered at a given point $\boldsymbol{z} \in \Omega$ and endowed with specified relative contrast $\beta_{z}$. The fixed domain $\mathcal{B}$ defines the shape of the trial anomaly, and can be assumed without loss of generality to verify $\int_{\mathcal{B}} \boldsymbol{x} \mathrm{d} V_{x}=\mathbf{0}$. We then set $D=B_{\varepsilon}=B_{\varepsilon}(\boldsymbol{z})$ in the functional (2.4). Denoting by $u_{\varepsilon}[g]:=u_{B_{\varepsilon}}[g]$ and $v_{\varepsilon}[g]:=u_{\varepsilon}[g]-u[g]$ the total and scattered fields arising in this situation, we then define the cost function $J[g](\varepsilon)=J[g](\varepsilon ; \boldsymbol{z})$ in terms of $\mathcal{J}[g]$ by

$$
J[g](\varepsilon)=\mathcal{J}[g]\left(B_{\varepsilon}\right)=\frac{1}{2} \int_{\Gamma}\left|v_{\varepsilon}[g]-v_{\text {obs }}[g]\right|^{2} \mathrm{~d} S
$$

The topological derivative (TD) $\mathcal{T}[g](\boldsymbol{z})$ of $J[g]$ at $\boldsymbol{z}$ is then defined as the leading coefficient in the expansion of $J(\varepsilon)-J(0)$ in powers of $\varepsilon$ :

$$
J[g](\varepsilon)=J[g](0)+\varepsilon^{3} \mathcal{T}[g](\boldsymbol{z})+o\left(\varepsilon^{3}\right)
$$

In view of (2.5) and recalling the error-free assumption on the measurement, $\mathcal{T}[g](\boldsymbol{z})$ can be evaluated by identification from

$$
-\operatorname{Re}\left\{\int_{\Gamma} \bar{v}_{\varepsilon}[g] v_{B}[g] \mathrm{d} S\right\}=\varepsilon^{3} \mathcal{T}[g](\boldsymbol{z})+o\left(\varepsilon^{3}\right) .
$$

Proceeding similarly, the TD $\mathcal{T}_{\Sigma}(\boldsymbol{z})$ of $J_{\Sigma}(\varepsilon):=\mathcal{J}_{\Sigma}\left(B_{\varepsilon}\right)$ is found from

$$
-\operatorname{Re}\left\{\sum_{m \geq 0} \int_{\Gamma} \bar{v}_{\varepsilon}\left[Y_{m}\right] v_{B}\left[Y_{m}\right] \mathrm{d} S\right\}=\varepsilon^{3} \mathcal{T}_{\Sigma}(\boldsymbol{z})+o\left(\varepsilon^{3}\right) .
$$

2.4. Representation of background and scattered fields. To derive the TDs arising from (2.6) and (2.7) in a form suitable for the analysis of their properties, it is convenient to introduce integral representations of the background and scattered fields. First, let the single-layer potential operator $\mathcal{S}: L^{2}(\Gamma) \rightarrow H^{1}(\Omega)$ be defined by

$$
\mathcal{S} \varphi(\boldsymbol{x})=\int_{\Gamma} G_{\kappa}(\boldsymbol{y}, \boldsymbol{x}) \varphi(\boldsymbol{y}) \mathrm{d} \boldsymbol{y} \quad \boldsymbol{x} \in \Omega,
$$


where $G_{\kappa}(\boldsymbol{y}, \boldsymbol{x})$ is the Neumann function for a unit point source $\boldsymbol{x}$ embedded in the background medium. The latter satisfies

$$
\left\{\begin{array}{rlrl}
-\left(\Delta+\kappa^{2}\right) G_{\kappa}(\cdot, \boldsymbol{x}) & =\delta(\boldsymbol{y}-\boldsymbol{x}) & & \text { in } \Omega \\
\partial_{n} G_{\kappa}(\cdot, \boldsymbol{x}) & =0 & & \text { on } \Gamma
\end{array} \quad \boldsymbol{x} \in \Omega,\right.
$$

( $\delta$ being the Dirac distribution) and has the following useful known properties:

Lemma 1. The Neumann function defined by problem (2.9) is real-valued. It verifies the symmetry properties

$$
G_{\kappa}(\boldsymbol{x}, \boldsymbol{y})=G_{\kappa}(\boldsymbol{y}, \boldsymbol{x}), \quad \boldsymbol{\nabla}_{1} G_{\kappa}(\boldsymbol{x}, \boldsymbol{y})=\boldsymbol{\nabla}_{2} G_{\kappa}(\boldsymbol{y}, \boldsymbol{x}) \quad \boldsymbol{x}, \boldsymbol{y} \in \mathbb{R}^{3}, \boldsymbol{x} \neq \boldsymbol{y},
$$

where $\nabla_{1} G_{\kappa}, \nabla_{2} G_{\kappa}$ are the gradients of $G_{\kappa}$ with respect to its first and second arguments.

The background field $u[g]$ solving $(2.1,2.3)$ is then given by

$$
u(x)=\mathcal{S} g(\boldsymbol{x}) .
$$

Then, let the volume potential operator $W_{\kappa}: L^{2}\left(\Omega ; \mathbb{C}^{3}\right) \rightarrow H^{1}(\Omega)$ be defined by

$$
W_{\kappa}[\boldsymbol{g}](\boldsymbol{x})=-\int_{\Omega} \nabla_{1} G_{\kappa}(\boldsymbol{y}, \boldsymbol{x}) \cdot \boldsymbol{g}(\boldsymbol{y}) \mathrm{d} \boldsymbol{y}
$$

for any vector density $\boldsymbol{q} \in L^{2}\left(\Omega ; \mathbb{C}^{3}\right)$. The scattered field for the true anomaly has the representation

$$
v_{B}(\boldsymbol{x} ; \boldsymbol{s})=W_{\kappa}[\boldsymbol{h}](\boldsymbol{x}) \quad \boldsymbol{x} \in B \cup(\Omega \backslash \bar{B}),
$$

where the density $\boldsymbol{h}=\beta \chi_{B} \boldsymbol{\nabla} u_{B}[g] \in L^{2}\left(B ; \mathbb{C}^{3}\right)$, supported in $B$, solves the singular volume integral equation (VIE)

$$
\left(\boldsymbol{I}-\beta \boldsymbol{\nabla} W_{\kappa}\right) \boldsymbol{h}(\boldsymbol{x})=\beta \boldsymbol{\nabla} u[g](\boldsymbol{x}) \quad \boldsymbol{x} \in B .
$$

The singular integral operator $\boldsymbol{I}-\beta \boldsymbol{\nabla} W_{\kappa}: L^{2}\left(B ; \mathbb{C}^{3}\right) \rightarrow L^{2}\left(B ; \mathbb{C}^{3}\right)$ is known to be invertible with bounded inverse (see e.g. Bonnet, 2017 for more details on singular VIE formulations). Solving equation (2.13), using (2.12) and recalling (2.10), we obtain

$$
v_{B}(\boldsymbol{x})=-\int_{B} \boldsymbol{\nabla}_{1} G_{\kappa}(\boldsymbol{y}, \boldsymbol{x}) \cdot\left[\boldsymbol{M}_{B} \boldsymbol{\nabla} S g\right](\boldsymbol{y}) \mathrm{d} \boldsymbol{y}
$$

where the solution operator $\boldsymbol{M}_{B}$, defined by $\boldsymbol{M}_{B} \boldsymbol{g}=\boldsymbol{h}$ with $\boldsymbol{h}$ solving $\left(\boldsymbol{I}-\beta \boldsymbol{\nabla} W_{\kappa}\right) \boldsymbol{h}=\beta \boldsymbol{g}$ for given $\boldsymbol{g} \in L^{2}\left(B ; \mathbb{C}^{3}\right)$, is easily verified to be given by

$$
\boldsymbol{M}_{B}=\left(\boldsymbol{I}-q \boldsymbol{R}_{\kappa}\right)^{-1} 2 q, \quad \text { with } q:=\beta /(\beta+2), \quad \boldsymbol{R}_{\kappa}:=\boldsymbol{I}+2 \boldsymbol{\nabla} W_{\kappa}
$$

where the modified material parameter $q \in(-1,1)$ will prove convenient. The real-valuedness and symmetry properties of the Neumann function (Lemma 1) imply that

Lemma 2. The solution operator $\boldsymbol{M}_{B}: L^{2}\left(B ; \mathbb{C}^{3}\right) \rightarrow L^{2}\left(B ; \mathbb{C}^{3}\right)$ is self-adjoint.

We also observe that the operators $\nabla \mathcal{S}: L^{2}(\Gamma) \rightarrow L^{2}\left(\Omega, \mathbb{C}^{3}\right)$ and $-W_{\kappa}: L^{2}\left(\Omega, \mathbb{C}^{3}\right) \rightarrow L^{2}(\Gamma)$ are mutual adjoints: for any $\varphi \in L^{2}(\Gamma)$ and $\boldsymbol{g} \in L^{2}\left(\Omega, \mathbb{C}^{3}\right)$, we have

$$
(\nabla \mathcal{S} \varphi, \boldsymbol{g})_{\Omega}=-\left(\varphi, W_{\kappa} \boldsymbol{g}\right)_{\Gamma}
$$

When $B=B_{\varepsilon}$, the scattered field for the trial anomaly $B_{\varepsilon}$ is given at any $\boldsymbol{x} \neq \boldsymbol{z}$ by the expansion

$$
v_{\varepsilon}(\boldsymbol{s})=-\varepsilon^{3} \nabla_{1} G_{\kappa}(\boldsymbol{z}, \boldsymbol{s}) \cdot \boldsymbol{M}_{\boldsymbol{z}} \cdot \boldsymbol{\nabla} S g(\boldsymbol{s})+o\left(\varepsilon^{3}\right),
$$

where $\boldsymbol{M}_{z}:=\boldsymbol{M}\left(\mathcal{B}, \beta_{z}\right) \in \mathbb{R}_{\mathrm{sym}}^{3 \times 3}$ is the (real, symmetric) polarization tensor of the normalized trial anomaly (Ammari et al., 2012; Cedio-Fengya et al., 1998). The expansion (2.14), and in particular its $O\left(\varepsilon^{3}\right)$ leading order, is well-known (see e.g. Ammari and Khelifi, 2003; Bendali et al., 2016; Cedio-Fengya et al., 1998; Hazard and Ramdani, 2004) and the VIE (2.13) provides a basis, among others, for its derivation and justification, as in Bonnet (2018). The tensor $\boldsymbol{M}_{\boldsymbol{z}}$, which can be understood as the limiting form of the solution operator $\boldsymbol{M}_{B}$ for $B=B_{\varepsilon}$ and is 
thus defined from the zero-frequency transmission problem where $\mathcal{B}$ is embedded in an infinite space and excited by a remote uniform gradient, is given by

$$
\boldsymbol{M}_{z} \cdot \boldsymbol{g}=2 q_{z} \int_{\mathcal{B}}\left(\boldsymbol{I}-q_{z} \boldsymbol{R}_{0}\right)^{-1} \boldsymbol{g} \mathrm{d} V \quad \text { for any } \boldsymbol{g} \in \mathbb{C}^{3}, \quad \text { with } q_{z}:=\beta_{z} /\left(\beta_{z}+2\right)
$$

where the (real, symmetric) $L^{2}\left(\mathcal{B} ; \mathbb{R}^{3}\right) \rightarrow L^{2}\left(\mathcal{B} ; \mathbb{R}^{3}\right)$ operator $\boldsymbol{R}_{0}$ is defined by $\boldsymbol{R}_{0}:=\boldsymbol{I}+2 \nabla W_{0}$, the volume potential $W_{0}$ being given by $(2.11)$ with $G_{\kappa}$ replaced with the zero-frequency fullspace fundamental solution $G_{0}(\boldsymbol{y}, \boldsymbol{x})=1 /(4 \pi|\boldsymbol{y}-\boldsymbol{x}|)$. Since $q_{z} \in(-1,1)$ and $\left\|\boldsymbol{R}_{0}\right\|<1$ (Bonnet, 2017 ), the operator $\boldsymbol{I}-q_{z} \boldsymbol{R}_{0}$ is real, symmetric positive definite (SPD). The matrix $\left(2 q_{z}\right)^{-1} \boldsymbol{M}_{z}$ is therefore also SPD, so has a Choleski square root $\boldsymbol{D}_{z}$, and we have

$$
\boldsymbol{M}_{z}=2 q_{z} \boldsymbol{D}_{z} \cdot \boldsymbol{D}_{z}^{\star}
$$

(with $\boldsymbol{D}_{z}=\sqrt{4 \pi /\left(3-q_{z}\right)} \boldsymbol{I}$ for a spherical trial anomaly).

2.5. Source-to-measurement operators and their factorization. As done customarily in inverse scattering studies, we introduce source-to-measurement $L^{2}(\Gamma) \rightarrow L^{2}(\Gamma)$ operators $F_{B}$ and $F_{z}$ associated with the true and trial scattered fields, such that

$$
\gamma v_{B}=F_{B} g, \quad \gamma v_{\varepsilon}=\varepsilon^{3} F_{z} g+o\left(\varepsilon^{3}\right),
$$

where $g$ is a given excitation applied on $\Gamma$ and $\gamma$ is the Dirichlet trace operator on $\Gamma$. Recalling that the Neumann function is real-valued, representations (2.14) and (2.17) yield

$$
F_{B}=-\boldsymbol{H}_{B}^{\star} \boldsymbol{M}_{B} \boldsymbol{H}_{B}, \quad F_{z}=-\boldsymbol{H}_{z}^{\star} \boldsymbol{M}_{z} \boldsymbol{H}_{z}
$$

where the operators $\boldsymbol{H}_{B}: L^{2}(\Gamma) \rightarrow L^{2}\left(B ; \mathbb{C}^{3}\right)$ and $\boldsymbol{H}_{z}: L^{2}(\Gamma) \rightarrow \mathbb{C}^{3}$ are defined by

$$
\boldsymbol{H}_{B} \varphi=\left.\nabla \mathcal{S} \varphi\right|_{B}, \quad \boldsymbol{H}_{z} \varphi=\nabla \mathcal{S} \varphi(\boldsymbol{z})
$$

in terms of the single-layer potential operator $(2.8)$ while $\boldsymbol{H}_{B}^{\star}: L^{2}\left(B ; \mathbb{C}^{3}\right) \rightarrow L^{2}(\Gamma)$ and $\boldsymbol{H}_{z}^{\star}$ : $\mathbb{C}^{3} \rightarrow L^{2}(\Gamma)$ are their respective adjoints, see (2.16). Lemma 2 implies that both $F_{B}$ and $F_{z}$ are self-adjoint $L^{2}(\Gamma) \rightarrow L^{2}(\Gamma)$ operators.

2.6. Topological derivatives. Taking advantage of the material collected in Sections 2.4 and 2.5, TDs can now be expressed from (2.6) and (2.7) in a form allowing their analysis as qualitative imaging functionals; this is the object of the following proposition, whose proof is given in Sec. 7.1.

Proposition 1. The single-measurement and full-measurement TDs arising from (2.6) and (2.7) are given by

$$
\begin{aligned}
\mathcal{T}[g](\boldsymbol{z}) & =-2 q_{z} \operatorname{Re}\left\{\int_{B}\left(\left[\boldsymbol{M}_{B} \boldsymbol{H}_{B} g\right](\boldsymbol{y}) \otimes\left(\boldsymbol{H}_{z} \bar{g}(\boldsymbol{z}) \cdot \boldsymbol{D}_{z}\right)\right):\left[\boldsymbol{K}(\boldsymbol{y}, \boldsymbol{z}) \cdot \boldsymbol{D}_{z}\right] d \boldsymbol{y}\right\} \\
\mathcal{T}_{\Sigma}(\boldsymbol{z}) & =-2 q_{z} \int_{B}\left[\boldsymbol{M}_{B}\left(\boldsymbol{K}(\boldsymbol{y}, \boldsymbol{z}) \cdot \boldsymbol{D}_{z}\right)\right]:\left[\boldsymbol{K}(\boldsymbol{y}, \boldsymbol{z}) \cdot \boldsymbol{D}_{z}\right] d \boldsymbol{y}
\end{aligned}
$$

where the (two-point, real, tensor-valued) function $\boldsymbol{K}$ is defined by

$$
\boldsymbol{K}(\boldsymbol{y}, \boldsymbol{z}):=\int_{\Gamma} \boldsymbol{\nabla}_{1} G_{\kappa}(\boldsymbol{y}, \boldsymbol{s}) \otimes \nabla_{1} G_{\kappa}(\boldsymbol{z}, \boldsymbol{s}) d \boldsymbol{s} .
$$

Remark 1. For multiple experiments (with applied excitations $g_{1}, \ldots, g_{M}$ ), we may simply set

$$
\mathcal{J}_{M}(D):=\sum_{m=1}^{M} \mathcal{J}\left[g_{m}\right](D), \quad \mathcal{T}_{M}(\boldsymbol{z}):=\sum_{m=1}^{M} \mathcal{T}\left[g_{m}\right](\boldsymbol{z}) .
$$

An additional single-measurement case of particular interest arises by observing that $F_{B}$ is compact (since the nonsingular integral operator $\boldsymbol{H}_{B}$ is), and hence has a countable complete orthonormal system with (real) eigenvalues $\lambda_{\ell}$ and eigenfunctions $\psi_{\ell} \in L^{2}(\Gamma)$. Hence, setting $g=\psi_{\ell}$ for some $\ell$, so that $g=-\lambda_{\ell}^{-1} F_{B} g=\lambda_{\ell}^{-1} \boldsymbol{H}_{B}^{\star} \boldsymbol{M}_{B} \boldsymbol{H}_{B} g$, we have 
Proposition 2. The eigenfunction-based TD is given by

$$
\mathcal{T}\left[\psi_{\ell}\right](\boldsymbol{z})=-\frac{2 q_{z}}{\lambda_{\ell}}\left|\int_{B} \boldsymbol{D}_{z}^{\star} \cdot \boldsymbol{K}^{\star}(\boldsymbol{y}, \boldsymbol{z}) \cdot\left[\boldsymbol{M}_{B} \boldsymbol{H}_{B} g\right](\boldsymbol{y}) d \boldsymbol{y}\right|^{2}
$$

with the two-point tensor function $\boldsymbol{K}$ again defined by (2.25).

Proof. The claimed expression results from setting $\boldsymbol{H}_{z} g=\lambda_{\ell}^{-1} \boldsymbol{H}_{z} \boldsymbol{H}_{B}^{\star} \boldsymbol{M}_{B} \boldsymbol{H}_{B} g$ in (7.1) and rearranging terms: we have

$$
\begin{aligned}
\mathcal{T}\left[\psi_{\ell}\right](\boldsymbol{z}) & =-\frac{1}{\lambda_{\ell}} \operatorname{Re}\left\{\left(\boldsymbol{M}_{B} \boldsymbol{H}_{B} g, \boldsymbol{H}_{B} \boldsymbol{H}_{z}^{\star} \boldsymbol{M}_{z} \boldsymbol{H}_{z} \boldsymbol{H}_{B}^{\star} \boldsymbol{M}_{B} \boldsymbol{H}_{B} g\right)_{B}\right\} \\
& =-\frac{2 q_{z}}{\lambda_{\ell}}\left(\boldsymbol{H}_{z} \boldsymbol{H}_{B}^{\star} \boldsymbol{M}_{B} \boldsymbol{H}_{B} g, \boldsymbol{M}_{z} \boldsymbol{H}_{z} \boldsymbol{H}_{B}^{\star} \boldsymbol{M}_{B} \boldsymbol{H}_{B} g\right),
\end{aligned}
$$

and (2.26) results from using the factorization (2.19) of $\boldsymbol{M}_{z}$ in the above formula and expressing $\boldsymbol{H}_{z} \boldsymbol{H}_{B}^{\star} \boldsymbol{M}_{B} \boldsymbol{H}_{B} g$ as an integral over $B$.

Remark 2. Formulas (2.23), (2.24) and (2.26) are not suitable for practical TD evaluations (e.g. for processing real experiments) since they involve the (unknown) anomaly to be identified. They are useful for analysis: as we will see next, they allow to establish results regarding properties of the TD functionals such as their sign and spatial behavior. Evaluation formulas suitable for applications, which express the TDs in terms of measurements and other known or assumed characteristics (domain, excitation, background medium, trial anomaly) by way of the background field, an adjoint field and the polarization tensor, are well known.

\section{Sign AND DECAY PRoperties of the topological DeRivatives}

We now use the expressions given in Propositions 1 and 2 to study the two components of the usual heuristic for TD imaging, namely the sign and spatial decay properties of the TD.

3.1. Sign properties. Those expressions first directly lead to the following theorem regarding the sign properties of the full-measurement and eigenfunction-based TDs:

Theorem 1. Let the relative material parameters $q$ and $q_{z}$ be as in (2.15) and (2.18).

(i) If the true scatterer $(B, \beta)$ and wave number $\kappa$ are such that

$$
\left\|q \boldsymbol{R}_{\kappa}\right\|=|q|\left\|\boldsymbol{R}_{\kappa}\right\|<1
$$

the full-measurement TD satisfies the sign condition

$$
\operatorname{sign}\left(\mathcal{T}_{\Sigma}(\boldsymbol{z})\right)=-\operatorname{sign}\left(q q_{z}\right) .
$$

(ii) Let $g=\psi_{\ell}$, where $\psi_{\ell}$ is an eigenfunction of $-F_{B}$ with (real) eigenvalue $\lambda_{\ell}$. Then, if $q \lambda_{\ell}>0$, we have

$$
\operatorname{sign}\left(\mathcal{T}\left[\psi_{\ell}\right](\boldsymbol{z})\right)=-\operatorname{sign}\left(q q_{z}\right)
$$

In particular, any eigenvalue of $-F_{B}$ satisfies $q \lambda_{\ell}>0$ if (3.1) is verified.

Proof. (i) In this case, we have

$$
\mathcal{T}_{\Sigma}(\boldsymbol{z})=-2 q_{z}\left(\boldsymbol{M}_{B} \boldsymbol{h}(\cdot, \boldsymbol{z}), \boldsymbol{h}(\cdot, \boldsymbol{z})\right)_{B},
$$

having set $\boldsymbol{h}(\boldsymbol{y}, \boldsymbol{z}):=\left[\boldsymbol{M}_{B}^{-1}\left(\boldsymbol{K}(\cdot, \boldsymbol{z}) \cdot \boldsymbol{D}_{z}\right)\right](\boldsymbol{y})$. If condition (3.1) is verified, $\boldsymbol{M}_{B}$ is self-adjoint sign-definite and has the same sign as $q$, which proves the sign property (3.1).

(ii) The claimed sign property is clear from the expression (2.26) of $\mathcal{T}\left[\psi_{\ell}\right](\boldsymbol{z})$. Moreover, for $g=\psi_{\ell}$, we have $\left(-F_{B} g, g\right)_{\Gamma}=\lambda_{\ell}(g, g)_{\Gamma}=\left(\boldsymbol{M}_{B} \boldsymbol{H}_{B} g, \boldsymbol{H}_{B} g\right)_{\Gamma}$. If condition (3.1) is verified, $\boldsymbol{M}_{B}$ is sign-definite (again) and we have $q \lambda_{\ell}>0$ for any eigenfunction $\psi_{\ell}$.

Condition (3.1) defines a class of "moderate" anomalies for which the validity of the sign heuristic is guaranteed (the moderate character depending on a combination of the operating frequency and the anomaly size and material contrast). Condition (3.1) ensures (for example) the convergence of iterated Born approximations. With the present notations, the more-restrictive standard Born approximation requires $\left\|q \boldsymbol{R}_{\kappa}\right\| \ll 1$. 


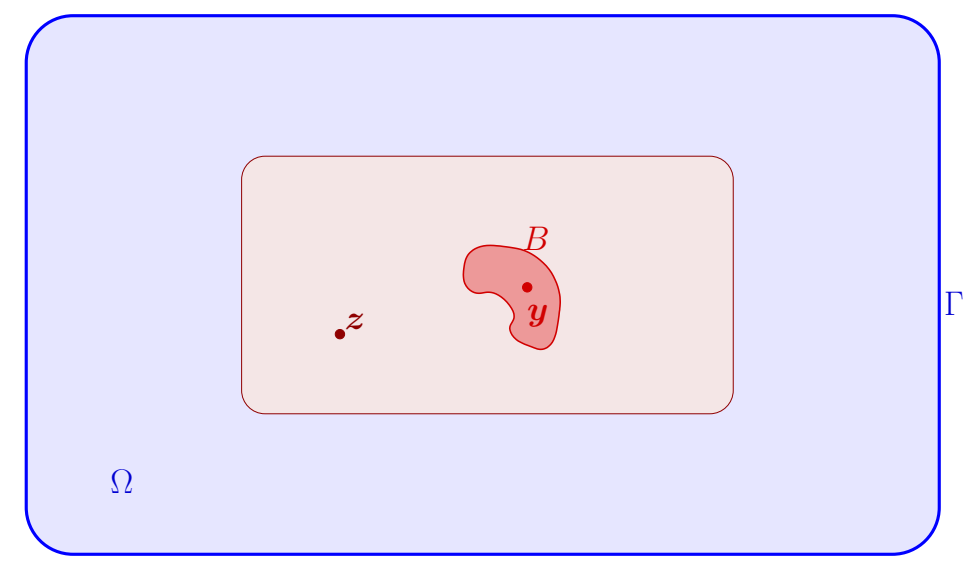

Figure 1. Scaling assumptions: the true anomaly $B$ (red) is much smaller than the probing region (shaded in blue), itself much smaller than the bounded propagation domain. Moreover, the distance of $B$ to the domain center is commensurate to its size.

3.2. Decay properties. As discussed by Ammari et al. (2012), Bellis et al. (2013), Bonnet and Cakoni (2019), to be used as a qualitative imaging function for the flaw $B, z \mapsto \mathcal{T}(\boldsymbol{z})$ should decay as $\boldsymbol{z}$ moves away from $B$ in addition to verifying the sign heuristic property. Here, we consider spatially-finite propagation media. We hence need to understand how $\mathcal{T}(\boldsymbol{z})$ decays for $\boldsymbol{z}$ "far" from $B$ and still remaining within a "reasonable" distance from $\Gamma$. The scaling hypotheses previously introduced in Bonnet and Cakoni (2019) are again adopted here, as they are well suited for studying the spatial decay properties of $z \mapsto \mathcal{T}(\boldsymbol{z})$ under these conditions. Let $\rho$ be a characteristic radius of $\Gamma$, for example such that $|\Gamma|=4 \pi \rho^{2}$. Taking a fixed $\boldsymbol{z}$ outside $B$, letting $\eta>0$ be a small parameter and choosing a constant $0<\alpha<1$, we assume that

$$
\left\{\begin{array}{l}
\text { (a) }|\boldsymbol{y}|=O(\operatorname{diam}(\mathrm{B})), \\
\text { (b) }|\boldsymbol{y}| / \rho=O(\eta), \\
\text { (c) }|\boldsymbol{y}-\boldsymbol{z}| / \rho=O\left(\eta^{\alpha}\right) \text { and }|\boldsymbol{y}| /|\boldsymbol{y}-\boldsymbol{z}|=O\left(\eta^{1-\alpha}\right) .
\end{array}\right.
$$

Loosely speaking, assumptions (3.2) mean that (a) the sought anomaly is located at a distance to the domain center (taken as the coordinate origin) commensurate with its linear size, (b) the anomaly and probing region are small relative to the characteristic radius of $\Gamma$, and (c) the sampling point $\boldsymbol{z}$ stays "far from" $B$ relative to its size while remaining "close to" $B$ relative to $\rho$ (Fig. 1). The following result about the spatial decay of topological derivatives, whose proof is given in Section 7.2, in fact assumes that $\Gamma$ is a sphere of radius $\rho$ (whereas the decay results of Sec. 5.2 hold for more general domains).

Theorem 2. Let $\Gamma$ be the sphere of radius $\rho$. Let the true anomaly $(B, \beta)$ and the sampling points $\boldsymbol{z}$ obey the scaling assumptions (3.2) with the dimensionless parameters $\alpha, \eta$ such that $0<\alpha<1$ and $0<\eta \ll 1$. Then:

$$
\begin{aligned}
\mathcal{T}[g](\boldsymbol{z}) & =O\left(\left[(\kappa \operatorname{dist}(\boldsymbol{z}, B))^{-1}+(\kappa \operatorname{dist}(-\boldsymbol{z}, B))^{-1}\right]\right) \\
\mathcal{T}_{\Sigma}(\boldsymbol{z}) & =O\left(\left[(\kappa \operatorname{dist}(\boldsymbol{z}, B))^{-1}+(\kappa \operatorname{dist}(-\boldsymbol{z}, B))^{-1}\right]^{2}\right) \\
\mathcal{T}\left[\psi_{\ell}\right](\boldsymbol{z}) & =O\left(\left[(\kappa \operatorname{dist}(\boldsymbol{z}, B))^{-1}+(\kappa \operatorname{dist}(-\boldsymbol{z}, B))^{-1}\right]^{2}\right)
\end{aligned}
$$

3.3. Discussion. The results of Theorems 1 and 2 exhibit many similarities with corresponding results from earlier studies involving either infinite propagation media or anomalies of small diameter. Condition (3.1) of Theorem 1 is identical to that appearing in the corresponding result of Bonnet and Cakoni (2019), except for the fact that $\boldsymbol{R}_{\kappa}$ is defined here in terms of the Neumann function. The spatial decay rates given in Theorem 1 are, likewise, identical to those found for their respective contexts in previous studies. However, Theorem 2 indicates that 
$\boldsymbol{z} \mapsto \mathcal{T}(\boldsymbol{z})$ decays only when $\boldsymbol{z}$ moves away from both $B$ and $-B$. This evidences a potentially significant degradation in spatial identification capability compared to earlier results where the decay is shown to occur as $\boldsymbol{z}$ moves away from $B$.

To remedy the unsatisfactory spatial behavior of the TD associated with standard $L^{2}$ cost functionals, we propose to apply in the present context the idea introduced by Ammari et al. (2012) whereby measurements are post-processed by application of a suitable integral operator. In addition, we express the background field as an incoming single-layer potential defined in the full space (a variation on an idea used in Bonnet and Cakoni, 2019). This results in a modified objective functional, and a correspondingly modified TD; importantly, thanks for the second treatment, the relevant source-to-measurement operators are still symmetric, allowing to preserve the structure of the analysis expressions of the TDs considered in this work.

The modified objective functionals and TDs are set up next in Section 4. Then, the counterparts of Theorems 1 and 2 are established in Section 5, where the modified TDs are in particular shown to have the expected (and desired) spatial behavior.

\section{Modified objective functional And topological DeRIVATiVes}

Let $G_{\kappa}^{\infty}(\boldsymbol{y}-\boldsymbol{x})$ be the fundamental solution for the infinite background medium, i.e. the outgoing field created by a time-harmonic unit point source located at $\boldsymbol{x} \in \mathbb{R}^{3}$, given by

$$
G_{\kappa}^{\infty}(\boldsymbol{y}-\boldsymbol{x}):=\frac{1}{4 \pi} \frac{e^{\mathrm{i} \kappa|\boldsymbol{y}-\boldsymbol{x}|}}{|\boldsymbol{y}-\boldsymbol{x}|} .
$$

and satisfying $-\left(\Delta+\kappa^{2}\right) G_{\kappa}^{\infty}(\cdot-\boldsymbol{x})=\delta(\cdot-\boldsymbol{x})$ in $\mathbb{R}^{3} \backslash\{\boldsymbol{x}\}$ together with the outgoing Sommerfeld radiation condition at infinity. Let $\mathcal{S}_{\infty}$ be the single-layer potential for the Helmholtz equation in $\mathbb{R}^{3}$ :

$$
\mathcal{S}_{\infty} \varphi(\boldsymbol{x})=\int_{\Gamma} G_{\kappa}(\boldsymbol{y}-\boldsymbol{x}) \varphi(\boldsymbol{y}) \mathrm{d} S(\boldsymbol{y}), \quad \boldsymbol{x} \in \mathbb{R}^{3} \backslash \Gamma,
$$

where $\varphi \in L^{2}(\Gamma)$. Let the integral operator $E$ and its $L^{2}(\Gamma)$-adjoint $E^{\star}$ be defined by

$$
\begin{array}{rlrl}
E \varphi(\boldsymbol{x}) & =\frac{1}{2} \varphi(\boldsymbol{x})+\int_{\Gamma} \partial_{\boldsymbol{n}(\boldsymbol{y})} G_{\kappa}^{\infty}(\boldsymbol{y}-\boldsymbol{x}) \varphi(\boldsymbol{y}) \mathrm{d} S(\boldsymbol{y}), & & \boldsymbol{x} \in \Gamma, \\
E^{\star} \varphi(\boldsymbol{x})=\frac{1}{2} \varphi(\boldsymbol{x})+\int_{\Gamma} \partial_{\boldsymbol{n}(\boldsymbol{x})} \overline{G_{\kappa}^{\infty}(\boldsymbol{y}-\boldsymbol{x})} \varphi(\boldsymbol{y}) \mathrm{d} S(\boldsymbol{y}), & \boldsymbol{x} \in \Gamma .
\end{array}
$$

Lemma 3. (i) The fundamental solution $G_{\kappa}^{\infty}$ and the Neumann function $G_{\kappa}$ defined by problem (2.9) are related by the identity

$$
\left[E G_{\kappa}(\cdot, \boldsymbol{z})\right](\boldsymbol{x})=G_{\kappa}^{\infty}(\boldsymbol{x}-\boldsymbol{z}), \quad \boldsymbol{x} \in \Gamma, \boldsymbol{z} \in \Omega .
$$

(ii) The background field $u=S g$ is equivalently given in $\Omega$ by

$$
u(\boldsymbol{x})=\mathcal{S} g(\boldsymbol{x})=\overline{\mathcal{S}_{\infty}} g_{\infty}(\boldsymbol{x})
$$

where, for given $g \in L^{2}(\Gamma)$, the density $g_{\infty} \in L^{2}(\Gamma)$ solves the integral equation

$$
E^{\star} g_{\infty}(\boldsymbol{x})=g(\boldsymbol{x}) \quad \boldsymbol{x} \in \Gamma
$$

(iii) We have $E \boldsymbol{H}_{B}^{\star}=\boldsymbol{H}_{B}^{\infty, \star}$ and $E \boldsymbol{H}_{z}^{\star}=\boldsymbol{H}_{z}^{\infty, \star}$, where the operators $\boldsymbol{H}_{B}^{\infty}: L^{2}(\Gamma) \rightarrow$ $L^{2}\left(B ; \mathbb{C}^{3}\right)$ and $\boldsymbol{H}_{z}^{\infty}: L^{2}(\Gamma) \rightarrow \mathbb{C}^{3}$ are defined by

$$
\boldsymbol{H}_{B}^{\infty} \varphi=\left.\overline{\nabla \mathcal{S}_{\infty}} \varphi\right|_{B}, \quad \boldsymbol{H}_{z}^{\infty} \varphi=\overline{\nabla \mathcal{S}_{\infty}} \varphi(\boldsymbol{z}) .
$$

Now, recalling that the (error-free) measurement $\gamma v_{B}$ is related to the excitation $g$ by $\gamma v_{B}=F_{B} g$, with the source-to-measurement operator $F_{B}$ defined by (2.21a), we use Lemma 3 to evaluate $E v_{B}$ and express $g$ in terms of $g_{\infty}$, to obtain

$$
E v_{B}\left[E^{\star} g_{\infty}\right]=E F_{B} E^{\star} g_{\infty}=F_{B}^{\infty} g_{\infty}, \quad F_{B}^{\infty}:=-\boldsymbol{H}_{B}^{\infty, \star} \boldsymbol{M}_{B} \boldsymbol{H}_{B}^{\infty} .
$$

The same operations performed on $v_{\varepsilon}=\varepsilon^{3} F_{z} g+o\left(\varepsilon^{3}\right)$ for the trial scattered field, with $F_{z}$ defined by $(2.21 \mathrm{~b})$, yield

$$
E F_{z}\left[g_{\infty}\right]=E F_{z} E^{\star} g_{\infty}=F_{z}^{\infty} g_{\infty}, \quad F_{z}^{\infty}:=-\boldsymbol{H}_{z}^{\infty, \star} \boldsymbol{M}_{z} \boldsymbol{H}_{z}^{\infty} .
$$


Remark 3. The modified source-to-measurement operators $F_{B}^{\infty}, F_{z}^{\infty}$ combine two existing ideas. First, applying $E$ to $F_{B} g=-F_{B}^{\infty} g_{\infty}=\boldsymbol{H}_{B}^{\star}\left(\boldsymbol{M}_{B} \boldsymbol{H}_{B} g\right)$ converts the field emitted in $\Omega$ by the secondary source $\boldsymbol{M}_{B} \boldsymbol{H}_{B} g$ associated with the scattering by the true anomaly into that emitted in an infinite medium by the same secondary source (and similarly for the asymptotic scattering model $F_{z} g$ ); such 'post-processing' of the output was introduced in Ammari et al. (2012). Second, setting $g=E^{\star} g_{\infty}$ achieves the conjugation $\mathcal{S} g=\overline{\mathcal{S}_{\infty}} g_{\infty} ;$ a similarly-motivated (but not identical) operation applied to the output $v_{B}$ was proposed in Bonnet and Cakoni (2019). The latter treatment helps remedy the lack of symmetry of the standard near-field source-to-measurement operator, an issue commonly arising with factorization methods in inverse scattering, see e.g. Audibert (2015), Audibert and Haddar (2017), Kirsch and Grinberg (2008). The resulting modified operators $F_{B}^{\infty}, F_{z}^{\infty}$ are compact and self-adjoint, like $F_{B}$ and $F_{z}$.

With the foregoing definitions, we introduce the modified form

$$
\mathcal{J}_{E}\left[g_{\infty}\right](D):=\frac{1}{2} \int_{\Gamma}\left|\left(E v_{D}\left[E^{\star} g_{\infty}\right]-E v_{\text {obs }}\left[E^{\star} g_{\infty}\right]\right)\right|^{2} \mathrm{~d} \boldsymbol{s}
$$

of the objective functional (2.4), which corresponds to the imaging functional (3.1) of Ammari et al. (2012) where in addition the excitation $g$ is converted to $g_{\infty}\left(\right.$ through $\left.g=E^{\star} g_{\infty}\right)$. Similarly, the modified form of the full-measurement cost functional $\mathcal{J}_{\Sigma}(D)$ is

$$
\mathcal{J}_{E, \Sigma}(D):=\sum_{m \geq 0} \frac{1}{2} \int_{\Gamma}\left|E v_{D}\left[E^{\star} Y_{m}\right]-E v_{\mathrm{obs}}\left[E^{\star} Y_{m}\right]\right|^{2} \mathrm{~d} S .
$$

Upon sampling the medium by means of trial anomalies $B_{\varepsilon}(\boldsymbol{z})$ as in Section 2.3, we set $J_{E}(\varepsilon):=$ $\mathcal{J}_{E}\left(B_{\varepsilon}\right)$ and $J_{E, \Sigma}(\varepsilon):=\mathcal{J}_{E, \Sigma}\left(B_{\varepsilon}\right)$. The TD of $J_{E}$ at $\boldsymbol{z}$ is then found again by identification:

$$
\begin{aligned}
J_{E}(\varepsilon)=-\operatorname{Re}\left\{\int_{\Gamma} \overline{E v_{\varepsilon}\left[g_{\infty}\right]} E v_{B}\left[g_{\infty}\right] \mathrm{d} S\right\}+ & o\left(\left\|v_{\varepsilon}\right\|_{\Gamma}\right)=\varepsilon^{3} \mathcal{T}_{E}\left[g_{\infty}\right](\boldsymbol{z})+o\left(\varepsilon^{3}\right), \\
& \text { with } \mathcal{T}_{E}\left[g_{\infty}\right](\boldsymbol{z})=-\operatorname{Re}\left\{\left(F_{B}^{\infty} g_{\infty}, F_{z}^{\infty} g_{\infty}\right)_{\Gamma}\right\},
\end{aligned}
$$

and similarly for $J_{E, \Sigma}$. Analysis expressions for the TDs corresponding to the modified singlemeasurement objective functional (4.2) and its full-measurement and eigenfunction-based versions are found as in Section 2.6 with $F_{B}, F_{z}$ replaced with $F_{B}^{\infty}$ and $F_{z}^{\infty}$, and we have:

Proposition 3. The single-measurement, full-measurement and eigenfunction-based modified TDs are given by

$$
\begin{aligned}
\mathcal{T}_{E}\left[g_{\infty}\right](\boldsymbol{z}) & =-2 q_{z} \operatorname{Re}\left\{\int_{B}\left(\left[\boldsymbol{M}_{B} \boldsymbol{H}_{B}^{\infty} g_{\infty}\right](\boldsymbol{y}) \otimes\left(\boldsymbol{H}_{z}^{\infty} \bar{g}(\boldsymbol{z}) \cdot \boldsymbol{D}_{z}\right)\right):\left[\boldsymbol{K}_{\infty}(\boldsymbol{y}, \boldsymbol{z}) \cdot \boldsymbol{D}_{z}\right] d \boldsymbol{y}\right\}(4.3) \\
\mathcal{T}_{E, \Sigma}(\boldsymbol{z}) & =-2 q_{z} \int_{B}\left[\boldsymbol{M}_{B}\left(\boldsymbol{K}_{\infty}(\boldsymbol{y}, \boldsymbol{z}) \cdot \boldsymbol{D}_{z}\right)\right]:\left[\boldsymbol{K}_{\infty}(\boldsymbol{y}, \boldsymbol{z}) \cdot \boldsymbol{D}_{z}\right] d \boldsymbol{y} \\
\mathcal{T}_{E}\left[\psi_{\ell}\right](\boldsymbol{z}) & =-\frac{2 q_{z}}{\lambda_{\ell}}\left|\int_{B} \boldsymbol{D}_{z}^{\star} \cdot \boldsymbol{K}_{\infty}^{\star}(\boldsymbol{y}, \boldsymbol{z}) \cdot\left[\boldsymbol{M}_{B} \boldsymbol{H}_{B}^{\infty} g\right](\boldsymbol{y}) d \boldsymbol{y}\right|^{2} \cdot
\end{aligned}
$$

where the (two-point, complex, tensor-valued) function $\boldsymbol{K}_{\infty}$ is defined by

$$
\boldsymbol{K}_{\infty}(\boldsymbol{y}, \boldsymbol{z}):=\int_{\Gamma} \boldsymbol{\nabla} G_{\kappa}^{\infty}(\boldsymbol{y}, \boldsymbol{s}) \otimes \overline{\boldsymbol{\nabla} G_{\kappa}^{\infty}(\boldsymbol{z}, \boldsymbol{s})} d \boldsymbol{s} .
$$

\section{Sign AND DECAY PROPERTIES OF The topological DeRivatives}

We now use the expressions of the modified TDs given in Proposition 3 to study their sign and decay properties.

5.1. Sign properties. The expressions given in Proposition 3 make it clear that the arguments underpinning Theorem 1 again apply to the modified TDs, which therefore have the same sign properties as their original counterparts:

Theorem 3. Let the relative material parameters $q$ and $q_{z}$ be again as in (2.15) and (2.18). 
(i) If the true scatterer $(B, \beta)$ and wave number $\kappa$ verify condition (3.1) of Theorem 1 , the full-measurement modified TD satisfies the sign condition

$$
\operatorname{sign}\left(\mathcal{T}_{E, \Sigma}(\boldsymbol{z})\right)=-\operatorname{sign}\left(q q_{z}\right) .
$$

(ii) Let $g=\psi_{\ell}$, where $\psi_{\ell}$ is an eigenfunction of $-F_{B}^{\infty}$ with (real) eigenvalue $\lambda_{\ell}$. Then, if $q \lambda_{\ell}>0$, we have

$$
\operatorname{sign}\left(\mathcal{T}_{E}\left[\psi_{\ell}\right](\boldsymbol{z})\right)=-\operatorname{sign}\left(q q_{z}\right) .
$$

In particular, any eigenvalue of $-F_{B}^{\infty}$ satisfies $q \lambda_{\ell}>0$ if (3.1) is verified.

5.2. Decay properties. The decay properties of the modified TDs given in Proposition 3 are now studied. As in Section 3.2 (and by adaptation of the approach of Bonnet and Cakoni, 2019 to the present context), this analysis requires determining the behavior of $\boldsymbol{K}_{\infty}(\boldsymbol{y}, \boldsymbol{z})$ as $\boldsymbol{z}$ moves away from $\boldsymbol{y}$. For this, we adopt again the scaling hypotheses (3.2), and rely on the "far field" approximation of $\boldsymbol{K}_{\infty}(\boldsymbol{y}, \boldsymbol{z})$ previously derived in Bonnet and Cakoni (2019), which retains the terms of order $O\left(\eta^{0}\right)$ and $O\left(\eta^{\alpha}\right)$ as $\eta \rightarrow 0$. This approximation is given in the following lemma, which is a rearranged version of eq. (33) in Bonnet and Cakoni (2019):

Lemma 4. Under the geometrical scaling hypotheses (3.2), the tensor function $\boldsymbol{K}_{\infty}(\boldsymbol{y}, \boldsymbol{z})$ defined by (2.25) admits the far-field expansion

$$
\begin{aligned}
\boldsymbol{K}_{\infty}(\boldsymbol{z}, \boldsymbol{y})= & \int_{\Gamma}\left\{e^{\mathrm{i} \kappa c|\boldsymbol{z}-\boldsymbol{y}|} A(\boldsymbol{s})\right. \\
& \left.\quad+e^{\mathrm{i} \kappa c|\boldsymbol{z}-\boldsymbol{y}|}\left[B(\boldsymbol{s} ; \hat{\boldsymbol{z}})+\frac{1}{2} \mathrm{i} \kappa|\boldsymbol{y}-\boldsymbol{z}|\left(1-c^{2}\right) C(\boldsymbol{s})\right] \frac{|\boldsymbol{y}-\boldsymbol{z}|}{\rho}\right\} d \boldsymbol{s}+o\left(\eta^{\alpha}\right)
\end{aligned}
$$

where $|\boldsymbol{y}-\boldsymbol{z}| / \rho=O\left(\eta^{\alpha}\right)($ see $(3.2)), c:=\hat{\boldsymbol{s}} \cdot \widehat{(\boldsymbol{y - z})}$ and with

$$
\begin{array}{rr}
A(\boldsymbol{s}) & =\frac{1+\kappa^{2}|\boldsymbol{s}|^{2}}{16 \pi^{2}|\boldsymbol{s}|^{2}} \hat{\boldsymbol{s}} \otimes \hat{\boldsymbol{s}}, \quad C(\boldsymbol{s})=\frac{\rho\left(1+\kappa^{2}|\boldsymbol{s}|^{2}\right)}{16 \pi^{2}|\boldsymbol{s}|^{3}} \hat{\boldsymbol{s}} \otimes \hat{\boldsymbol{s}}, \\
B(\boldsymbol{s} ; \hat{\boldsymbol{z}}) & =-\frac{\rho\left(1+\kappa^{2}|\boldsymbol{s}|^{2}\right)}{16 \pi^{2}|\boldsymbol{s}|^{3}} \hat{\boldsymbol{z}} \otimes \hat{\boldsymbol{s}}-\frac{c \rho\left(3+\mathrm{i} \kappa|\boldsymbol{s}|+2 \kappa^{2}|\boldsymbol{s}|^{2}\right)}{16 \pi^{2}|\boldsymbol{s}|^{3}} \hat{\boldsymbol{s}} \otimes \hat{\boldsymbol{s}}
\end{array}
$$

The $O\left(\eta^{0}\right)$ terms are those arising in the usual leading-order far-field expansion of $\nabla G_{\kappa}^{\infty}$.

To carry out further the analysis of the behavior of $\boldsymbol{K}_{\infty}(\boldsymbol{y}, \boldsymbol{z})$, we assume the surface $\Gamma$ to be smooth and star-shaped with respect to the coordinate origin, so that it can be parametrized onto the unit sphere $\hat{S}$ via a mapping of the form $s=\rho \boldsymbol{\sigma}(\hat{\boldsymbol{s}})$, where $\rho$ is a characteristic radius of $\Gamma$ and $\hat{\boldsymbol{s}} \mapsto \boldsymbol{\sigma}(\hat{\boldsymbol{s}})$ is a $C^{2}\left(\hat{S} ; \mathbb{R}^{3}\right)$ fixed (i.e. scale-independent) function. We further set $\hat{\boldsymbol{s}}=\hat{\boldsymbol{s}}(\varphi, c)=\sqrt{1-c^{2}}\left(\cos \varphi \boldsymbol{e}_{x}+\sin \varphi \boldsymbol{e}_{y}\right)+c \boldsymbol{e}_{z}$ with $c \in[-1,1], \varphi \in[0,2 \pi]$ and where $\left(\boldsymbol{e}_{x}, \boldsymbol{e}_{y}, \boldsymbol{e}_{z}\right)$ is a Cartesian orthonormal frame such that $\boldsymbol{e}_{z}=\widehat{\boldsymbol{y}-\boldsymbol{z}}$. The surface differential $\mathrm{d} \boldsymbol{s}$ then takes the form $\mathrm{d} \boldsymbol{s}=\rho^{2} J(\hat{\boldsymbol{s}}) \mathrm{d} c \mathrm{~d} \varphi$.

We proceed with the evaluation of the approximation (5.1) of $\boldsymbol{K}_{\infty}(\boldsymbol{z}, \boldsymbol{y})$, whose integrand is a sum of three terms. In the first two terms, the exponential factor is expressed using the Jacobi-Anger expansion

$$
e^{\mathrm{i} \kappa c|\boldsymbol{z}-\boldsymbol{y}|}=\sum_{n \geq 0}(2 n+1) \mathrm{i}^{-n} j_{n}(\kappa|\boldsymbol{z}-\boldsymbol{y}|) P_{n}(c)
$$

(where $P_{n}$ is the Legendre polynomial of degree $n$ ). In the third term, we instead use the following expansion of the product $\left(1-c^{2}\right) e^{\mathrm{i} \kappa c|\boldsymbol{z}-\boldsymbol{y}|}$ (proved in Sec. 7.4), based on the JacobiAnger expansion (5.2) and properties of the Legendre polynomials:

\section{Lemma 5.}

$$
\left(1-c^{2}\right) e^{\mathrm{i} \kappa c|\boldsymbol{z}-\boldsymbol{y}|}=\sum_{n \geq 0}(2 n+1) \mathrm{i}^{-n}\left\{\frac{n(n-1)}{\kappa^{2}|\boldsymbol{z}-\boldsymbol{y}|^{2}} j_{n}(\kappa|\boldsymbol{z}-\boldsymbol{y}|)+\frac{2}{\kappa|\boldsymbol{z}-\boldsymbol{y}|} j_{n+1}(\kappa|\boldsymbol{z}-\boldsymbol{y}|)\right\} P_{n}(c)
$$


Performing these substitutions in (5.1), expressing the resulting series as a sum of multiples of $P_{n}(c)$ and converting all integrals into integrals over the unit sphere by using the mapping $\boldsymbol{s}=\boldsymbol{\sigma}(\hat{\boldsymbol{s}})$, we arrive at the following result:

Proposition 4. The asymptotic approximation (5.1) of $\boldsymbol{K}_{\infty}(\boldsymbol{y}, \boldsymbol{z})$ admits the expression

$$
\begin{aligned}
\boldsymbol{K}_{\infty}(\boldsymbol{z}, \boldsymbol{y}) & =\sum_{n \geq 0} \rho^{2}(2 n+1) \mathrm{i}^{-n}\left\{\mathrm{~A}_{n} j_{n}(\kappa|\boldsymbol{z}-\boldsymbol{y}|)\right. \\
& \left.\left.+\left[\left(\mathrm{B}_{n}(\hat{\boldsymbol{z}})+\mathrm{iC}_{n} \frac{n(n-1)}{2 \kappa|\boldsymbol{z}-\boldsymbol{y}|}\right) j_{n}(\kappa|\boldsymbol{z}-\boldsymbol{y}|)+\mathrm{iC}_{n} j_{n-1}(\kappa|\boldsymbol{z}-\boldsymbol{y}|)\right)\right] \frac{|\boldsymbol{z}-\boldsymbol{y}|}{\rho}\right\}+o\left(\eta^{\alpha}\right)
\end{aligned}
$$

where

$$
\begin{aligned}
\mathrm{A}_{n} & :=\int_{-1}^{1}\left\{\int_{0}^{2 \pi} A(\boldsymbol{\sigma}(\hat{\boldsymbol{s}})) J(c, \varphi) d \varphi\right\} P_{n}(c) d c \\
\mathrm{~B}_{n}(\hat{\boldsymbol{z}}) & :=\int_{-1}^{1}\left\{\int_{0}^{2 \pi} \frac{1}{|\boldsymbol{\sigma}(\hat{\boldsymbol{s}})|} B(\boldsymbol{\sigma}(\hat{\boldsymbol{s}}) ; \hat{\boldsymbol{z}}) J(c, \varphi) d \varphi\right\} P_{n}(c) d c \\
\mathrm{C}_{n} & :=\frac{\mathrm{i} \kappa}{2} \int_{-1}^{1}\left\{\int_{0}^{2 \pi} \frac{1}{|\boldsymbol{\sigma}(\hat{\boldsymbol{s}})|} C(\boldsymbol{\sigma}(\hat{\boldsymbol{s}})) J(c, \varphi) d \varphi\right\}\left(1-c^{2}\right) P_{n}(c) d c
\end{aligned}
$$

For fixed $\rho$ satisfying $(3.2 \mathrm{~b})$, i.e. large enough with respect to the anomaly $B$, the behavior $j_{n}(t)=O\left(|t|^{-1}\right)$ for $t \rightarrow \infty$ of the spherical Bessel functions implies that $\boldsymbol{K}_{\infty}(\boldsymbol{z}, \boldsymbol{y})=O((\kappa \mid \boldsymbol{y}-$ $\boldsymbol{z} \mid)^{-1}$ ) for $\boldsymbol{y} \in B$ and $\boldsymbol{z}$ far from $B$ while satisfying (3.2c). Since the solution operator $\boldsymbol{M}_{B}$ is bounded, applying Proposition 4 together with the Cauchy-Schwarz inequality to the modified TDs of Proposition 3 readily yields the following spatial decay properties:

Theorem 4. Let $\Gamma$ satisfy the assumptions given after Lemma 4. Let the true anomaly $(B, \beta)$ and the sampling points $\boldsymbol{z}$ obey the scaling assumptions (3.2) with the dimensionless parameters $\alpha, \eta$ such that $0<\alpha<1$ and $0<\eta \ll 1$. Then:

$$
\begin{aligned}
\mathcal{T}_{E}\left[g_{\infty}\right](\boldsymbol{z}) & =O\left((\kappa \operatorname{dist}(\boldsymbol{z}, B))^{-1}\right) \\
\mathcal{T}_{E, \Sigma}(\boldsymbol{z}) & =O\left((\kappa \operatorname{dist}(\boldsymbol{z}, B))^{-2}\right) \\
\mathcal{T}_{E}\left[\psi_{\ell}\right](\boldsymbol{z}) & =O\left((\kappa \operatorname{dist}(\boldsymbol{z}, B))^{-2}\right)
\end{aligned}
$$

Remark 4. The decay properties of Theorem 4 do not depend on the choice of $\alpha \in(0,1)$ in the scaling assumption (3.2c), which quantifies the fact that $\rho$ is much larger than the sampling region. The term of (5.1) which could possibly have affected the $\alpha$-independent decay of $\boldsymbol{K}_{\infty}(\boldsymbol{z}, \boldsymbol{y})$ is that in $C(\boldsymbol{s})$ due to the factor $|\boldsymbol{y}-\boldsymbol{z}|$. Lemma 5 shows that the latter factor disappears, and for that reason plays an important role in obtaining the final spatial decay result.

\section{Discussion, AdDitional situations}

6.1. Discussion. The rates of spatial decay in the present setting (bounded propagation medium, separation of scales according to (3.2)) are the same as those previously found for unbounded media and either refraction-index anomalies and far-field measurements (Bellis et al., 2013) or mass-density anomalies and measurements at finite distance (Bonnet and Cakoni, 2019). Likewise, conditions ensuring the validity of the sign heuristic are of the same nature there and here. The analysis formulas (2.23) and (4.3) do not readily allow to assess the sign of the single-measurement TDs for arbitrarily chosen excitations.

As in Bellis et al. (2013) for the far-field case, the eigenfunction-based (standard or modified) TDs $\mathcal{T}\left[\psi_{\ell}\right]$ and $\mathcal{T}_{E}\left[\psi_{\ell}\right]$ have a spatial decay that is sharper than that of the single-measurement TDs $\mathcal{T}[g], \mathcal{T}_{E}[g]$, and identical in rate to that of the full-measurement TDs $\mathcal{T}_{\Sigma}$ and $\mathcal{T}_{E, \Sigma}$. This property is reminiscent of the focusing ability of time-reversal methods (Burkard et al., 2013; Hazard and Ramdani, 2004; Prada and Fink, 1994), which also exploit eigenfunctions of the source-to-measurement operator. Moreover, the eigenfunction-based TDs have the correct sign (relative toi the imaging heuristic) provided the sign of the eigenvalue $\lambda_{\ell}$ is suitable, a condition less restrictive than the operator-norm bound (3.1). 
6.2. Anisotropic anomaly. We discuss briefly the case where the sought anomaly has an anisotropic relative contrast characterized by a real symmetric matrix $\boldsymbol{\beta} \in \mathbb{R}_{\mathrm{sym}}^{3 \times 3}$ ( $\boldsymbol{\beta}$ may be indefinite, with eigenvalues greater than -1$)$. The wave $u_{B}$ then satisfies $\operatorname{div}\left(\boldsymbol{\beta} \cdot \boldsymbol{\nabla} u_{B}\right)+\kappa^{2} u_{B}=0$ inside $B$ and $\left.\partial_{n} u_{B}\right|_{+}=\left.\boldsymbol{n} \cdot \boldsymbol{\beta} \cdot \boldsymbol{\nabla} u_{B}\right|_{-}$across $\partial B$, while the governing VIE (2.13) becomes

$$
\left(\boldsymbol{I}-\boldsymbol{\beta} \cdot \boldsymbol{\nabla} W_{\kappa}\right) \boldsymbol{h}(\boldsymbol{x})=\boldsymbol{\beta} \cdot \boldsymbol{\nabla} u[g](\boldsymbol{x}) \quad \boldsymbol{x} \in B .
$$

Letting $\boldsymbol{q}:=(\boldsymbol{\beta}+2 \boldsymbol{I})^{-1} \cdot \boldsymbol{\beta}$ be the anisotropic counterpart of the modified material parameter $q$ introduced in (2.15), it is easy to show that the solution operator $\boldsymbol{M}_{B}$ is given in the anisotropic case by $\boldsymbol{M}_{B}=2\left(\boldsymbol{I}-\boldsymbol{q} \cdot \boldsymbol{R}_{\kappa}\right)^{-1} \cdot \boldsymbol{q}$.

If $\boldsymbol{\beta}$ (and hence $\boldsymbol{q}$ ) is sign-definite, there exists a real matrix $\boldsymbol{d} \in \mathbb{R}^{3 \times 3}$ such that $\boldsymbol{q}=\sigma \boldsymbol{d}^{\mathrm{T}} \cdot \boldsymbol{d}$, where $\sigma= \pm 1$ according to the sign of $\boldsymbol{q}$. In this case, $\boldsymbol{M}_{B}$ can be recast in symmetric form as

$$
\boldsymbol{M}_{B}=2 \boldsymbol{d}^{\mathrm{T}} \cdot\left(\boldsymbol{I}-\boldsymbol{d} \cdot \boldsymbol{R}_{\kappa} \cdot \boldsymbol{d}^{\mathrm{T}}\right)^{-1} \cdot \boldsymbol{d} .
$$

Consequently, analysis expressions of $\mathcal{T}_{\Sigma}, \mathcal{T}_{E, \Sigma}, \mathcal{T}\left[\psi_{\ell}\right]$ and $\mathcal{T}_{E}\left[\psi_{\ell}\right]$ have the same structure as their isotropic counterparts of Propositions 1, 2 and 3, and the sign heuristic results of Theorems 1 and 3 remain valid with $q q_{z}$ replaced with $\sigma q_{z}$ and the condition (3.1) recast as $\left\|\boldsymbol{d} \cdot \boldsymbol{R}_{\kappa} \cdot \boldsymbol{d}^{\mathrm{T}}\right\|<1$. Moreover, the decay properties given in Theorems 2 and 4 for the isotropic case remain valid. A similar analysis was made in Bonnet and Cakoni (2019) for sources and receivers embedded in an unbounded propagation medium, with similar results.

6.3. Refraction-index anomaly. Here we consider the alternative, simpler case where anomalies are characterized by a refraction-index contrast $\eta$, so that the total field $u_{B}$ propagates according to

$$
\begin{gathered}
-\left(\Delta+\kappa^{2}\right) u=0 \quad \text { in } \Omega \backslash \bar{B}, \quad-\left(\Delta+(\eta+1) \kappa^{2}\right) u=0 \quad \text { in } B \\
\left.u\right|_{+}=\left.u\right|_{-},\left.\quad \partial_{n} u\right|_{+}=\left.\partial_{n} u\right|_{-} \quad \text { on } \partial B
\end{gathered}
$$

The above problem can be recast as the Lippmann-Schwinger VIE governing the scalar density $h:=\eta u_{B} \in H^{1}(B)$ :

$$
\left(I-\kappa^{2} \eta V_{\kappa}\right) h(\boldsymbol{x})=\eta u[g](\boldsymbol{x}) \quad \boldsymbol{x} \in B,
$$

where $V_{\kappa}$ is the weakly-singular volume potential operator defined by

$$
V_{\kappa} h(\boldsymbol{x})=\int_{B} G_{\kappa}(\boldsymbol{y}, \boldsymbol{x}) h(\boldsymbol{y}) \mathrm{d} \boldsymbol{y} .
$$

The medium is then sampled with trial refraction-index anomalies $B_{\varepsilon}$ with specified contrast $\eta_{z}$. The (symmetric) source-to-measurement operators $F_{B}$ and $F_{z}$ such that $\gamma v_{B}=F_{B} g$ and $\gamma v_{\varepsilon}=\varepsilon^{3}\left[F_{z} g+o(1)\right]$ are then readily found to be given by

$$
F_{B}=H_{B}^{\star} M_{B} H_{B}, \quad F_{z}=\eta_{z}|\mathcal{B}| H_{z}^{\star} H_{z}
$$

where the operators $H_{B}: L^{2}(\Gamma) \rightarrow H^{1}(B)$ and $H_{z}: L^{2}(\Gamma) \rightarrow \mathbb{C}$ are defined by $H_{B} \varphi=\left.\mathcal{S} \varphi\right|_{B}$ and $H_{z} \varphi=\mathcal{S} \varphi(\boldsymbol{z})$ in terms of the single-layer potential operator (2.8), and the solution operator $M_{B}$ is given by $M_{B}=\left(I-\kappa^{2} \eta V_{\kappa}\right)^{-1} \kappa^{2} \eta$ (i.e. achieves $\kappa^{2} h=M_{B}(\eta u)$ with $h$ solving (6.1)). The asymptotic solution operator $M_{z}$ is here simply the scalar multiplication by $\eta_{z}|\mathcal{B}|$. Then, processing the sources and measurements as in Section 4, we again set the modified source-tomeasurement operators $F_{B}^{\infty}:=H_{B}^{\infty, \star} M_{B} H_{B}^{\infty}$ and $F_{z}:=\eta_{z}|\mathcal{B}| H_{z}^{\infty, \star} H_{z}^{\infty}$ with $H_{B}^{\infty} \varphi=\left.\mathcal{S}_{\infty} \varphi\right|_{B}$ and $H_{z}^{\infty} \varphi=\mathcal{S}_{\infty} \varphi(\boldsymbol{z})$. The TDs arising from (2.6) and (2.7) are as a result found to be given by

$$
\begin{gathered}
\mathcal{T}[g](\boldsymbol{z})=-2 \eta_{z}|\mathcal{B}| \operatorname{Re}\left\{H_{z} \bar{g}(\boldsymbol{z}) \int_{B}\left[M_{B} H_{B} g\right](\boldsymbol{y}) L(\boldsymbol{y}, \boldsymbol{z}) \mathrm{d} \boldsymbol{y}\right\} \\
\mathcal{T}_{\Sigma}(\boldsymbol{z})=-2 \eta_{z}|\mathcal{B}| \int_{B}\left[M_{B} L(\cdot, \boldsymbol{z})\right](\boldsymbol{y}) L(\boldsymbol{y}, \boldsymbol{z}) \mathrm{d} \boldsymbol{y} \\
\mathcal{T}\left[\psi_{\ell}\right](\boldsymbol{z})=-\frac{2 \eta_{z}|\mathcal{B}|}{\lambda_{\ell}}\left|\int_{B} L(\boldsymbol{y}, \boldsymbol{z})\left[M_{B} H_{B} g\right](\boldsymbol{y}) \mathrm{d} \boldsymbol{y}\right|^{2} .
\end{gathered}
$$


where $\psi_{\ell}$ is an eigenfunction of $F_{B}$ with eigenvalue $\lambda_{\ell}$ and the scalar real function $L$ is defined by (7.2), while the corresponding modified TDs are given by

$$
\begin{aligned}
\mathcal{T}_{E}\left[g_{\infty}\right](\boldsymbol{z}) & =-2 \eta_{z}|\mathcal{B}| \operatorname{Re}\left\{H_{z}^{\infty} \bar{g}(\boldsymbol{z}) \int_{B}\left[M_{B} H_{B}^{\infty} g\right](\boldsymbol{y}) L_{\infty}(\boldsymbol{y}, \boldsymbol{z}) \mathrm{d} \boldsymbol{y}\right\} \\
\mathcal{T}_{E, \Sigma}(\boldsymbol{z}) & =-2 \eta_{z}|\mathcal{B}| \int_{B}\left[M_{B} L_{\infty}(\cdot, \boldsymbol{z})\right](\boldsymbol{y}) L_{\infty}(\boldsymbol{y}, \boldsymbol{z}) \mathrm{d} \boldsymbol{y} \\
\mathcal{T}_{E}\left[\psi_{\ell}\right](\boldsymbol{z}) & =-\frac{2 \eta_{z}|\mathcal{B}|}{\lambda_{\ell}}\left|\int_{B} L_{\infty}(\boldsymbol{y}, \boldsymbol{z})\left[M_{B} H_{B}^{\infty} g\right](\boldsymbol{y}) \mathrm{d} \boldsymbol{y}\right|^{2} .
\end{aligned}
$$

where $\psi_{\ell}$ is an eigenfunction of $F_{B}^{\infty}$ with eigenvalue $\lambda_{\ell}$ and the scalar complex function $L_{\infty}$ is defined by

$$
L_{\infty}(\boldsymbol{y}, \boldsymbol{z}):=\int_{\Gamma} G_{\kappa}^{\infty}(\boldsymbol{s}, \boldsymbol{y}) \overline{G_{\kappa}^{\infty}(\boldsymbol{s}, \boldsymbol{z})} \mathrm{d} \boldsymbol{s}
$$

From here on, sign properties analogous to those of Theorems 1 and 3 are obtained, with $q q_{z}$ replaced with $\eta \eta_{z}$ and the condition (3.1) recast as $\left\|\kappa^{2} \eta V_{\kappa}\right\|<1$, while the behavior of $L(\boldsymbol{y}, \boldsymbol{z})$ and $L_{\infty}(\boldsymbol{y}, \boldsymbol{z})$ produce again the decay properties of Theorems 2 and 4 . In particular, the spatial behavior of the standard TDs is as before found to be degraded by "echoes" of the true anomaly.

6.4. Inhomogeneous anomaly. Consider the case where the anomaly is characterized by a spatially-varying relative mass density contrast $\beta \in L^{\infty}(B)$ such that $\beta \geq \beta_{0}$ in $B$ for some $\beta_{0}>-1$. The contrast is moreover assumed to be sign-definite, i.e. $\beta=\sigma|\beta|$, where the contrast $\operatorname{sign} \sigma= \pm 1$ is constant in $B$, and we then also have $q=\sigma|q|$. The symmetric factorizations (2.21) and (4.1) of the source-to-measurement operators $F_{B}$ and $F_{B}^{\infty}$ are still available by recasting the solution operator $M_{B}$ as

$$
M_{B}=\sigma \sqrt{2|q|}\left(\boldsymbol{I}-\sigma \sqrt{|q|} \boldsymbol{R}_{\kappa} \sqrt{|q|}\right)^{-1} \sqrt{2|q|} .
$$

Theorems 1 and 3 on sign properties remain valid with $\operatorname{sign}\left(q q_{z}\right)$ replaced with $\operatorname{sign}\left(\sigma q_{z}\right)$ and condition (3.1) with $\sup _{\boldsymbol{x} \in B}|q(\boldsymbol{x})|\left\|\boldsymbol{R}_{\kappa}\right\|<1$. Moreover, theorems 2 and 4 on decay properties still hold.

\section{Proofs}

7.1. Proof of Proposition 1. First, inserting expressions (2.20) in (2.6) and expanding $F_{B}, F_{z}$ by means of (2.21), the single-measurement TD is obtained as

$$
\begin{aligned}
\mathcal{T}[g](\boldsymbol{z}) & =-\operatorname{Re}\left\{\left(F_{B} g, F_{z} g\right)_{\Gamma}\right\} \\
& =-\operatorname{Re}\left\{\left(\boldsymbol{M}_{B} \boldsymbol{H}_{B} g, \boldsymbol{H}_{B} \boldsymbol{H}_{z}^{\star} \boldsymbol{M}_{z} \boldsymbol{H}_{z} g\right)_{B}\right\} .
\end{aligned}
$$

We then observe that, from definitions (2.22), we have

$$
\boldsymbol{H}_{B} \boldsymbol{H}_{z}^{\star} \boldsymbol{f}=\boldsymbol{K}(\boldsymbol{y}, \boldsymbol{z}) \cdot \boldsymbol{f}
$$

for any $\boldsymbol{f} \in \mathbb{C}^{3}$, with $\boldsymbol{K}(\boldsymbol{y}, \boldsymbol{z})$ given by (2.25). Using this identity and the factorization (2.19) of $\boldsymbol{M}_{z}$ in (7.1) and rearranging terms by means of tensor algebra yields (2.23).

For the full-measurement TD, we have

$$
\mathcal{T}_{\Sigma}(\boldsymbol{z})=-\operatorname{Re}\left\{\int_{B}\left(\sum_{m \geq 0}\left[\boldsymbol{M}_{B} \boldsymbol{H}_{B} Y_{m}\right](\boldsymbol{y}) \otimes\left(\boldsymbol{H}_{B} \bar{Y}_{m}(\boldsymbol{z}) \cdot \boldsymbol{D}_{z}\right)\right):\left[\boldsymbol{K}(\boldsymbol{y}, \boldsymbol{z}) \cdot \boldsymbol{D}_{z}\right] \mathrm{d} \boldsymbol{y}\right\}
$$


by summing appropriate expressions of the form (2.23). Besides, noting that $\boldsymbol{H}_{B} Y_{m}(\boldsymbol{y})=$ $\int_{\Gamma} \boldsymbol{\nabla}_{1} G_{\kappa}(\boldsymbol{y}, \boldsymbol{s}) Y_{m}(\boldsymbol{s}) \mathrm{d} \boldsymbol{s}=\overline{\left(\boldsymbol{\nabla}_{1} G_{\kappa}(\boldsymbol{y}, \cdot), Y_{m}\right)_{\Gamma}}$ and $\boldsymbol{H}_{B} \bar{Y}_{m}(\boldsymbol{z})=\left(\boldsymbol{\nabla}_{1} G_{\kappa}(\boldsymbol{z}, \cdot), Y_{m}\right)_{\Gamma}$, we deduce

$$
\begin{aligned}
\boldsymbol{K}(\boldsymbol{y}, \boldsymbol{z}) & =\int_{\Gamma}\left[\sum_{m \geq 0}\left(\boldsymbol{\nabla}_{1} G_{\kappa}(\boldsymbol{y}, \cdot), \bar{Y}_{m}\right)_{\Gamma} \bar{Y}_{m}(\boldsymbol{s})\right] \otimes\left[\sum_{n \geq 0}\left(\boldsymbol{\nabla}_{1} G_{\kappa}(\boldsymbol{z}, \cdot), Y_{n}\right)_{\Gamma} Y_{n}(\boldsymbol{s})\right] \mathrm{d} \boldsymbol{s} \\
& =\sum_{m \geq 0} \frac{\left(\boldsymbol{\nabla}_{1} G_{\kappa}(\boldsymbol{y}, \cdot), Y_{m}\right)_{\Gamma}}{} \otimes\left(\boldsymbol{\nabla}_{1} G_{\kappa}(\boldsymbol{z}, \cdot), Y_{m}\right)_{\Gamma} \\
& =\sum_{m \geq 0} \boldsymbol{H}_{B} Y_{m}(\cdot) \otimes \boldsymbol{H}_{B} \bar{Y}_{m}(\boldsymbol{z})
\end{aligned}
$$

since (by assumption) the $Y_{m}$ form a $L^{2}(\Gamma)$-orthonormal Hilbert basis of $L^{2}(\Gamma)$. This allows to recast $\mathcal{T}_{\Sigma}(\boldsymbol{z})$ in the sought form $(2.24)$, which is more concise and structure-revealing.

7.2. Proof of Theorem 2. All three estimates claimed in the theorem are found by studying the behavior of $\boldsymbol{K}(\boldsymbol{y}, \boldsymbol{z})$ under the scaling assumptions (3.2). We first observe that

$$
\boldsymbol{K}(\boldsymbol{y}, \boldsymbol{z})=\nabla_{1} \nabla_{2} L(\boldsymbol{y}, \boldsymbol{z}), \quad L(\boldsymbol{y}, \boldsymbol{z}):=\int_{\Gamma} G_{\kappa}(\boldsymbol{s}, \boldsymbol{y}) G_{\kappa}(\boldsymbol{s}, \boldsymbol{z}) \mathrm{d} \boldsymbol{s},
$$

then take advantage of the fact that the Neumann function $G_{\kappa}$ is known analytically when $\Gamma$ is a sphere, by means of classical separation of variable methods:

$$
G_{\kappa}(\boldsymbol{s}, \boldsymbol{z})=\kappa \sum_{n \geq 0} \sum_{|m| \leq n} \overline{Y_{n}^{m}(\hat{\boldsymbol{z}})} Y_{n}^{m}(\hat{\boldsymbol{s}}) j_{n}(\kappa|\boldsymbol{z}|)\left(\frac{y_{n}^{\prime}(\kappa \rho)}{j_{n}^{\prime}(\kappa \rho)} j_{n}(\kappa|\boldsymbol{s}|)-y_{n}(\kappa|\boldsymbol{s}|)\right),
$$

where $j_{n}$ and $y_{n}$ are the spherical Bessel functions of the first and second kinds, respectively. Then, since the spherical harmonics $Y_{n}^{m}$ are $L^{2}(\Gamma)$-orthonormal functions, we have

$$
L(\boldsymbol{y}, \boldsymbol{z})=\frac{1}{(\kappa \rho)^{2}} \sum_{n \geq 0} \sum_{|m| \leq n} Y_{n}^{m}(\hat{\boldsymbol{y}}) \overline{Y_{n}^{m}(\hat{\boldsymbol{z}})} \frac{j_{n}(\kappa|\boldsymbol{y}|)}{j_{n}^{\prime}(\kappa \rho)} \frac{j_{n}(\kappa|\boldsymbol{z}|)}{j_{n}^{\prime}(\kappa \rho)}
$$

For reasons given at the end of this proof, the general term of the above series decays rapidly (more than exponentially fast in $n$ ) for $n$ larger than $n_{1}=O\left(\frac{1}{2} e \kappa \operatorname{diam}(B)\right)$, the arguments being kept fixed, so that only the terms for $n \leq n_{1}$ are in practice relevant. For such values of $n$ and in view of the scaling assumptions (3.2), we have $\kappa \rho \gg n$ so that $j_{n}^{\prime}(\kappa \rho)$ can be approximated using the well-known large-argument asymptotics of the spherical Bessel functions (available e.g. in (Olver et al., 2010, Sec. 10.52(ii))):

$$
\frac{1}{\left(t j_{n}^{\prime}(t)\right)^{2}}=q_{n}(t)+O\left(\frac{1}{t}\right), \quad q_{n}(t):=\frac{1}{\sin ^{2}\left(t-\frac{1}{2}(n+1) \pi\right)}
$$

where in fact we have

$$
q_{2 n}(t)=q_{0}(t)=\frac{1}{\sin ^{2}\left(t-\frac{\pi}{2}\right)}, \quad q_{2 n+1}(t)=q_{1}(t)=\frac{1}{\cos ^{2}\left(t-\frac{\pi}{2}\right)}
$$

Using this in (7.3) with $t=\kappa|\boldsymbol{y}-\boldsymbol{z}|$, and setting $q_{0}=r_{0}+r_{1}$ and $q_{1}=r_{0}-r_{1}$, in $L(\boldsymbol{y}, \boldsymbol{z})$ we obtain

$$
\begin{aligned}
L(\boldsymbol{y}, \boldsymbol{z}) & =\sum_{n \geq 0} \sum_{|m| \leq n}\left[r_{0}(\kappa \rho)+(-1)^{n} r_{1}(\kappa \rho)\right] \overline{Y_{n}^{m}(\hat{\boldsymbol{z}})} Y_{n}^{m}(\hat{\boldsymbol{y}}) j_{n}(\kappa|\boldsymbol{z}|) j_{n}(\kappa|\boldsymbol{y}|)+O\left(\frac{1}{\kappa \rho}\right) \\
& =r_{0}(\kappa \rho) j_{0}(\kappa|\boldsymbol{z}-\boldsymbol{y}|)+r_{1}(\kappa \rho) j_{0}(\kappa|\boldsymbol{z}+\boldsymbol{y}|)+O\left(\frac{1}{\kappa \rho}\right)
\end{aligned}
$$

In view of (7.5), the same method of proof can be applied, with suitable alterations, to $\boldsymbol{K}(\boldsymbol{y}, \boldsymbol{z})$ as the spherical Bessel functions and their derivatives obey similar large-argument and large-order asymptotics.

We finish with a qualitative explanation of why using (7.4) is valid. The spherical Bessel functions in (7.3) involve three arguments, which are such that $\kappa|\boldsymbol{y}| \ll \kappa|\boldsymbol{z}| \ll \kappa \rho$ under the scaling assumptions (3.2). Accordingly, with $n_{1}$ as above and setting $n_{2}=O(\kappa|\boldsymbol{z}|)=O\left(\eta^{\alpha-1} n_{1}\right)$ 
and $n_{3}=O(\kappa \rho)=O\left(\eta^{-1} n_{1}\right)$ (so that in particular we have $\left.n_{1} \ll n_{2} \ll n_{3}\right)$, the general

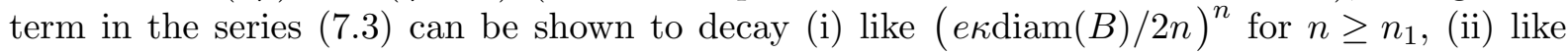
$\left(e^{2} \kappa^{2}|\boldsymbol{z}| \operatorname{diam}(B) / 4 n^{2}\right)^{n}$ for $n \geq n_{2}$, and (iii) like $(\sqrt{|\boldsymbol{z}| \operatorname{diam}(B)} / \kappa \rho)^{2 n} / n^{2}$ for $n \geq n_{3}$, by invoking the large-order asymptotics of $j_{n}(\kappa|\boldsymbol{y}|), j_{n}(\kappa|\boldsymbol{z}|)$ and $\kappa \rho j_{n}^{\prime}(\kappa \rho)$, respectively (Olver et al., 2010, Sec. 10.19)). Hence, the sum (7.3) can in practice be truncated at $n=n_{1}$. Hence (7.4) is used with $t=\kappa \rho \gg n$, so that the underlying large-argument asymptotics is valid. This (abbreviated and informal) argument could be made rigorous, at the cost of a rather longer and more-technical proof.

7.3. Proof of Lemma 3. (i) The complementary Neumann function $G_{\kappa}^{\mathrm{c}}(\boldsymbol{y}, \boldsymbol{z}):=G_{\kappa}(\boldsymbol{y}-$ $\boldsymbol{z})-G_{\kappa}^{\infty}(\boldsymbol{y}, \boldsymbol{z})$ is non-singular in $\Omega$; it verifies the Helmholtz equation in $\Omega$ and $\partial_{\boldsymbol{n}} G_{\kappa}^{\mathrm{c}}(\cdot, \boldsymbol{z})+$ $\partial_{\boldsymbol{n}} G_{\kappa}^{\mathrm{c}}(\cdot, \boldsymbol{z})=0$ on $\Gamma$. It therefore satisfies the interior boundary integral equation (BIE)

$$
\left[E G_{\kappa}^{\mathrm{c}}(\cdot, \boldsymbol{z})\right](\boldsymbol{x})+\left[S_{\infty} \partial_{\boldsymbol{n}} G_{\kappa}^{\infty}(\cdot, \boldsymbol{z})\right](\boldsymbol{x})=0 \quad \boldsymbol{x} \in \Gamma
$$

where $S_{\infty}:=\gamma \mathcal{S}_{\infty}$ is the single-layer integral operator. Moreover, $G_{\kappa}^{\infty}(\boldsymbol{y}, \boldsymbol{z})$ is a radiating solution of the Helmholtz equation in $\mathbb{R}^{3} \backslash \bar{\Omega}$, so satisfies the exterior BIE

$$
-G_{\kappa}^{\infty}(\boldsymbol{x}, \boldsymbol{z})+\left[E G_{\kappa}^{\infty}(\cdot, \boldsymbol{z})\right](\boldsymbol{x})-\left[S_{\infty} \partial_{\boldsymbol{n}} G_{\kappa}^{\infty}(\cdot, \boldsymbol{z})\right](\boldsymbol{x})=0 \quad \boldsymbol{x} \in \Gamma
$$

The claimed integral identity results from adding the two BIEs.

(ii) We seek $g_{\infty} \in L^{2}(\Gamma)$ such that $\mathcal{S} g=\overline{\mathcal{S}_{\infty}} g_{\infty}$ in $\Omega$ for given $g \in L^{2}(\Gamma)$. Taking the interior Neumann trace of this equality, we obtain the sought BIE

$$
g=E^{\star} g_{\infty} \text { on } \Gamma,
$$

by virtue of the definition of the Neumann function and the classical trace properties of layer potentials. This BIE is well-posed since by assumption $\kappa$ is not a Neumann eigenvalue of $-\Delta$ in $\Omega$.

(iii) Differentiating identity (i) with respect to $\boldsymbol{y} \in \Omega$ and invoking Lemma 1, we find

$$
\boldsymbol{\nabla} G_{\kappa}^{\infty}(\boldsymbol{y}-\boldsymbol{x})=\left[E \boldsymbol{\nabla}_{2} G_{\kappa}(\cdot, \boldsymbol{y})\right](\boldsymbol{x})=\left[E \boldsymbol{\nabla}_{1} G_{\kappa}(\boldsymbol{y}, \cdot)\right](\boldsymbol{x}) .
$$

Then, right-multiplying by $\boldsymbol{h}(\boldsymbol{y})$ and integrating over $\boldsymbol{y} \in B$ yields $\boldsymbol{H}_{B}^{\infty, \star} \boldsymbol{h}=E \boldsymbol{H}_{B}^{\star} \boldsymbol{h}$ for any $\boldsymbol{h}(\boldsymbol{y}) \in L^{2}\left(B, \mathbb{C}^{3}\right)$. The proof of $E \boldsymbol{H}_{z}^{\star}=\boldsymbol{H}_{z}^{\infty, \star}$ is similar.

7.4. Proof of Lemma (5). The lemma relies on the following identity expressing $\left(1-c^{2}\right) P_{n}(c)$ as a linear combination of Legendre polynomials:

Lemma 6. For any $c \in[-1,1]$ and $n \in \mathbb{N}$, we have

$$
(2 n+1)\left(1-c^{2}\right) P_{n}(c)=a_{n}\left[P_{n}(c)-P_{n-2}(c)\right]-a_{n+2}\left[P_{n+2}(c)-P_{n}(c)\right]
$$

where

$$
a_{n}=\frac{n(n-1)}{2 n-1}
$$

Proof. The identity follows from two consecutive applications to $c^{2} P_{n}(c)$ of the classical recurrence relation

$$
(2 m+1) c P_{m}(c)=(m+1) P_{m+1}(c)+m P_{m-1}(c) \quad m=0,1,2 \ldots
$$

verified by the Legendre polynomials, and subsequent rearrangement.

We then evaluate $\left(1-c^{2}\right) e^{\text {ict }}$ for arbitrary $t \in \mathbb{R}$ using the Jacobi-Anger expansion (5.2) and Lemma 6, which yields

$$
\begin{aligned}
\left(1-c^{2}\right) e^{\mathrm{i} c t} & =\sum_{n \geq 0}(2 n+1) \mathrm{i}^{-n} j_{n}(t)\left(1-c^{2}\right) P_{n}(c) \\
& =\sum_{n \geq 0} \mathrm{i}^{-n} j_{n}(t)\left\{a_{n}\left[P_{n}(c)-P_{n-2}(c)\right]-a_{n+2}\left[P_{n+2}(c)-P_{n}(c)\right]\right\},
\end{aligned}
$$


and rearrange the above sum as a sum of multiples of $P_{n}(c)$, to obtain

$$
\left(1-c^{2}\right) e^{\mathrm{i} c t}=\sum_{n \geq 0} \mathrm{i}^{-n} P_{n}(c)\left\{a_{n} j_{n-2}(t)+\left(a_{n}+a_{n+2}\right) j_{n}(t)+a_{n+2} j_{n+2}(t)\right\}
$$

(since $a_{0}=a_{1}=0$, the terms involving $j_{-2}(t)$ and $j_{-1}(t)$ vanish, as they must, in the above sum). We then apply the classical identity $\left(j_{m-1}+j_{m+1}\right)(t)=(2 m+1) j_{m}(t) / t$ (see e.g. Olver et al., 2010, Sec. 10.51.1) to recast the bracketed factors of the above sum as

$$
\begin{aligned}
a_{n} j_{n-2}(t) & +\left(a_{n}+a_{n+2}\right) j_{n}(t)+a_{n+2} j_{n+2}(t) \\
= & (2 n-1) a_{n} \frac{j_{n-1}(t)}{t}+(2 n+3) a_{n+2} \frac{j_{n+1}(t)}{t} \\
= & (2 n-1)(2 n+1) a_{n} \frac{j_{n}(t)}{t^{2}}+\left[(2 n+3) a_{n+2}-(2 n-1) a_{n}\right] \frac{j_{n+1}(t)}{t} \\
= & (2 n+1)\left\{n(n-1) \frac{j_{n}(t)}{t^{2}}+2 \frac{j_{n+1}(t)}{t}\right\}
\end{aligned}
$$

Substitution of the above formula into (7.6) therefores provides

$$
\left(1-c^{2}\right) e^{\mathrm{i} c t}=\sum_{n \geq 0}(2 n+1) \mathrm{i}^{-n}\left\{n(n-1) \frac{j_{n}(t)}{t^{2}}+2 \frac{j_{n+1}(t)}{t}\right\} P_{n}(c),
$$

whereupon Lemma 5 follows from the above sum with $t=\kappa|\boldsymbol{y}-\boldsymbol{z}|$.

\section{REFERENCES}

H. Ammari and A. Khelifi. Electromagnetic scattering by small dielectric inhomogeneities. J. Maths Pures Appl., 82:749-842, 2003.

H. Ammari, J. Garnier, V. Jugnon, and H. Kang. Stability and resolution analysis for a topological derivative based imaging functional. SIAM J. Contr. Opt., 50:48-76, 2012.

H. Ammari, E. Bretin, J. Garnier, H. Jing, W. Kang, and A. Wahab. Localization, stability, and resolution of topological derivative based imaging functionals in elasticity. SIAM J. Imag. Sci., 6:2174-2212, 2013.

L. Audibert. Qualitative methods for heterogeneous media. PhD thesis, Ecole Polytechnique, Palaiseau, France, 2015.

L. Audibert and H. Haddar. The generalized linear sampling method for limited aperture measurements. SIAM J. Imag. Sci., 10:845-870, 2017.

C. Bellis and M. Bonnet. Qualitative identification of cracks using 3d transient elastodynamic topological derivative: formulation and fe implementation. Comput. Meth. Appl. Mech. Engrg., 253:89-105, 2013.

C. Bellis, M. Bonnet, and F. Cakoni. Acoustic inverse scattering using topological derivative of far-field measurements-based $l^{2}$ cost functionals. Inverse Problems, 29:075012, 2013 . doi: http://dx.doi.org/10.1088/0266-5611/29/7/075012.

A. Bendali, P.-H. Cocquet, and S. Tordeux. Approximation by multipoles of the multiple acoustic scattering by small obstacles in three dimensions and application to the foldy theory of isotropic scattering. Arch. Ration. Mech. An., 219:1017-1059, 2016.

M. Bonnet. A modified volume integral equation for anisotropic elastic or conducting inhomogeneities. unconditional solvability by neumann series. J. Integral Eq. Appl., 29:271-295, 2017.

M. Bonnet. Inverse acoustic scattering using high-order topological derivatives of misfit functional. Inverse Probl. Imag., 12:921-953, 2018.

M. Bonnet and F. Cakoni. Analysis of topological derivative as a tool for qualitative identification. Inverse Problems, 35:104007, 2019.

M. Bonnet and B. B. Guzina. Sounding of finite solid bodies by way of topological derivative. Int. J. Numer. Meth. Engrg., 61:2344-2373, 2004.

C. Burkard, A. Minut, and K. Ramdani. Far-field model for time reversal and application to selective focusing on small dielectric inhomogeneities. Inverse Probl. Imag., 7:445-470, 2013.

F. Cakoni and D. Colton. A qualitative approach to inverse scattering theory. Springer-Verlag, 2014.

F. Cakoni, D. Colton, and H. Haddar. Inverse scattering theory and transmission eigenvalues. CBMS-NSF Regional Conference Series in Applied Mathematics. SIAM, 2016.

A. Carpio, T. G. Dimiduk, F. le Louër, and M. L. Rapún. When topological derivatives met regularized Gauss-Newton iterations in holographic 3D imaging. J. Comput. Phys., 388:224-251, 2019. 
J. Céa, S. Garreau, P. Guillaume, and M. Masmoudi. The shape and topological optimization connection. Comput. Meth. Appl. Mech. Engrg., 188:713-726, 2000.

D. J. Cedio-Fengya, S. Moskow, and M. Vogelius. Identification of conductivity imperfections of small diameter by boundary measurements. continuous dependence and computational reconstruction. Inverse Problems, 14:553-595, 1998.

S. Cogar. Analysis of a trace class stekloff eigenvalue problem arising in inverse scattering. SIAM J. Appl. Math., 80:881-905, 2020.

N. Dominguez, V. Gibiat, and Y. Esquerré. Time domain topological gradient and time reversal analogy: an inverse method for ultrasonic target detection. Wave Motion, 42:31-52, 2005.

H. A. Eschenauer, V. V. Kobelev, and A. Schumacher. Bubble method for topology and shape optimization of structures. Structural Optimization, 8:42-51, 1994.

B. B. Guzina and M. Bonnet. Small-inclusion asymptotic of misfit functionals for inverse problems in acoustics. Inverse Problems, 22:1761-1785, 2006.

B. B. Guzina and I. Chikichev. From imaging to material identification: a generalized concept of topological sensitivity. J. Mech. Phys. Solids, 55:245-279, 2007.

B. B Guzina and F. Pourahmadian. Why the high-frequency inverse scattering by topological sensitivity may work. Proc. Roy. Soc. A, 471:20150187, 2015.

C. Hazard and K. Ramdani. Selective acoustic focusing using time-harmonic reversal mirrors. SIAM J. Appl. Math., 64:1657-1676, 2004.

A. Kirsch and N. Grinberg. The factorization method for inverse problems. Oxford, 2008.

A. Laurain, M. Hintermüller, M. Freiberger, and H. Scharfetter. Topological sensitivity analysis in fluorescence optical tomography. Inverse Problems, 29:025003, 2013.

F. Le Louër and M.-L. Rapún. Topological sensitivity for solving inverse multiple scattering problems in three-dimensional electromagnetism. part i: One step method. SIAM J. Imag. Sci., 10:1291-1321, 2017.

A. A. Novotny, J. Sokołowski, and A. Żochowski. Topological derivatives of shape functionals. part i: theory in singularly perturbed geometrical domains. J. Optim. Th. \& Appl., 180:341-373, 2019a.

A. A. Novotny, J. Sokołowski, and A. Żochowski. Topological derivatives of shape functionals. part II: first-order method and applications. J. Optim. Th. ES Appl., 180:683-710, 2019b.

A. A. Novotny, J. Sokołowski, and A. Żochowski. Topological derivatives of shape functionals. part III: second-order method and applications. J. Optim. Th. \& Appl., 181:1-22, 2019c.

F. W. J. Olver, D. W. Lozier, R. F. Boisvert, and C. W. Clark, editors. NIST handbook of mathematical functions. Cambridge, 2010.

C. Prada and M. Fink. Eigenmodes of the time reversal operator: A solution to selective focusing in multiple-target media. Wave Motion, 20:151-163, 1994.

J. Sokolowski and A. Zochowski. On the topological derivative in shape optimization. SIAM J. Control Optim., 37:1251-1272, 1999.

A. Wahab. Stability and resolution analysis of topological derivative based localization of small electromagnetic inclusions. SIAM J. Imag. Sci., 8:1687-1717, 2015.

POEMS (CNRS, INRIA, ENSTA), ENSTA, 828 Boulevard des MaréChaux, 91120 Palaiseau, FranCe. Email address: mbonnet@ensta.fr 